\title{
Generating Series for Nested Bethe Vectors ${ }^{\star}$
}

\author{
Sergey KHOROSHKIN ${ }^{\dagger}$ and Stanislav PAKULIAK ${ }^{\ddagger \dagger}$ \\ $\dagger$ Institute of Theoretical \& Experimental Physics, 117259 Moscow, Russia \\ E-mail:khor@itep.ru \\ $¥$ Laboratory of Theoretical Physics, JINR, 141980 Dubna, Moscow Region, Russia \\ E-mail: pakuliak@theor.jinr.ru
}

Received September 14, 2008; Published online November 24, 2008

Original article is available at http://www.emis.de/journals/SIGMA/2008/081/

\begin{abstract}
We reformulate nested relations between off-shell $U_{q}\left(\widehat{\mathfrak{g l}}_{N}\right)$ Bethe vectors as a certain equation on generating series of strings of the composed $U_{q}\left(\widehat{\mathfrak{g l}}_{N}\right)$ currents. Using inversion of the generating series we find a new type of hierarchical relations between universal off-shell Bethe vectors, useful for a derivation of Bethe equation. As an example of application, we use these relations for a derivation of analytical Bethe ansatz equations [Arnaudon D. et al., Ann. Henri Poincaré 7 (2006), 1217-1268, math-ph/0512037] for the parameters of universal Bethe vectors of the algebra $U_{q}\left(\widehat{\mathfrak{g l}}_{2}\right)$.
\end{abstract}

Key words: Bethe ansatz; current algebras; quantum integrable models

2000 Mathematics Subject Classification: 17B37; 81R50

\section{Introduction}

Hierarchical (nested) Bethe ansatz (NBA) was designed [12] to solve quantum integrable models with $\mathfrak{g l}_{N}$ symmetries. The cornerstone of NBA is a procedure which relates Bethe vectors for the model with $\mathfrak{g l}_{N}$ symmetry to analogous objects with $\mathfrak{g l}_{N-1}$ symmetry. This hierarchical procedure is implicit, it allows to obtain Bethe equations for the parameters of the Bethe vectors while the explicit construction of these vectors itself remains rather non-trivial problem. Authors of the papers $[16,17]$ proposed a closed expression for off-shell Bethe vectors as matrix elements of the monodromy operator built of products of fundamental L-operators. However a calculation of this expression in every particular representation is still a nontrivial problem. Such a calculations was done in [18] on the level of the evaluation homomorphism of $U_{q}\left(\widehat{\mathfrak{g l}}_{N}\right) \rightarrow U_{q}\left(\mathfrak{g l}_{N}\right)$

The construction of $[16,17]$ yields the off-shell Bethe vectors in terms of matrix elements of the monodromy matrix which satisfies the corresponding quantum Yang-Baxter equation and generate a Borel subalgebra of quantum affine algebra $U_{q}\left(\widehat{\mathfrak{g l}}_{N}\right)$ [15] for trigonometric $R$-matrix or the Yangian $Y\left(\mathfrak{g l}_{N}\right)$ for the rational $R$-matrix. Those quantum affine algebras as well as doubles of Yangians possess another "new" realization introduced in [2]. In this realization the corresponding algebra is described in terms of generating series (currents) and an isomorphism between different realizations of these infinite-dimensional algebras was observed in [3]. Using this isomorphism one may try to look for the expressions for the universal off-shell Bethe vectors in terms of the modes of the currents. This program was realized in [9, 10], where explicit formulas for the off-shell Bethe vectors in terms of the currents were found. A significant part of this approach to the construction of Bethe vectors is a method of projection introduced in [7] and developed in the recent paper [6]. This method operates with projections of Borel subalgebra to its intersections with Borel subalgebras of a different type. It was proved in [6] that universal off-shell Bethe vectors can be identified with the projections of products of the

\footnotetext{
${ }^{\star}$ This paper is a contribution to the Special Issue on Kac-Moody Algebras and Applications. The full collection is available at http://www.emis.de/journals/SIGMA/Kac-Moody_algebras.html
} 
Drinfeld currents to the intersections of Borel subalgebras of different types. In the latter paper it was checked in a rather general setting that the Bethe vectors obtained from the projections of the currents satisfy the same comultiplication rule as the Bethe vectors constructed in terms of the fundamental L-operators. This approach was used in $[11,14]$ for further generalization of the results obtained in [18].

A deduction of general expressions for off-shell Bethe vectors [11, 14] is based on hierarchical relations between projections of products of the currents (see Proposition 4.2 in [11]). However these relations do not help in the investigation of the action of integrals of motions on off-shell Bethe vectors. The situation is quite different in classical approach of nested Bethe ansatz. The corresponding hierarchical relations allow to compute the action of integrals of motion and derive the Bethe equations [12, 13], but can be hardly used in the computation of the explicit expressions of Bethe vectors. This signifies an existence of two types of hierarchical relations for off-shell Bethe vectors and thus of two different presentations of them. The goal of this paper is to observe these two type of the hierarchical relations within the approach of the method of projections.

The new important objects which appear in the application of the method of projections to the investigation of the off-shell Bethe vectors are so called strings and their projections. Strings are special ordered products of composed current introduced in [4]. Our hierarchical relations express $U_{q}\left(\widehat{\mathfrak{g l}}_{N}\right)$ Bethe vectors via products of special strings and $U_{q}\left(\widehat{\mathfrak{g l}}_{N-1}\right)$ Bethe vectors. On the other hand the basic point of application of the method of projection is the ordered decomposition of the product of the currents. The factors of this decomposition are projections of the products of the currents (3.12). The crucial observation is that this decomposition and hierarchical relations for the opposite projections of the products of currents have a similar structure. Combinations of relations of these two different type can be used to obtain a new type hierarchical relations for off-shell Bethe vectors.

In order to solve the latter problem we collect all off-shell Bethe vectors into multi-variable generating series. We also introduce the generating series for the products of the currents and for the strings and their projections. We rewrite the hierarchical relations for $U_{q}\left(\widehat{\mathfrak{g l}}_{N}\right)$ Bethe vectors as a simple relation on the product of the generating series of the projections of the strings and the generating series of the $U_{q}\left(\widehat{\mathfrak{g l}}_{N-1}\right)$ Bethe vectors. A similar construction can be performed for off-shell Bethe vectors related to opposite Borel subalgebra. However the product entering into these relations is not usual. It contains a $q$-symmetrization with a special functional weight. This $\star$-product is associative and the generating series are invertible with respect to this product. Finally the new type of the hierarchical relations reduces to inversion of the generating series of opposite projections of the strings. This inversion is effectively performed. To do this we introduce some combinatorial language of tableaux filled by the Bethe parameters (see Section 4.5).

Applications of the new type hierarchical relations to the investigation of the properties of quantum integrals of motion are given in [8]. Here we demonstrate how they work in the simplest case of the universal $U_{q}\left(\widehat{\mathfrak{g l}}_{2}\right)$ Bethe vectors. As a result we get universal Bethe equations of the analytical Bethe ansatz [1]. In contrast to the usual Bethe equations these equations refer to Cartan currents instead of the eigenvalues of the diagonal elements of monodromy matrix on

highest weight vectors. In the Appendix we collect the basic defining relations for the $U_{q}\left(\widehat{\mathfrak{g l}}_{N}\right)$ composed currents.

\section{Generating series and $\star$-product}

\section{$2.1 \quad$ A $q$-symmetrization}

Let $\bar{t}=\left\{t_{1}, \ldots, t_{n}\right\}$ be a set of formal variables. Let $G(\bar{t})$ be a Laurent series taking values in $U_{q}\left(\widehat{\mathfrak{g l}}_{N}\right)$. Consider the permutation group $S_{n}$ and its action on the formal series of $n$ variables 
$\bar{t}=\left\{t_{1}, \ldots, t_{n}\right\}$ defined for the elementary transpositions $\sigma_{i, i+1}$ as follows

$$
\pi\left(\sigma_{i, i+1}\right) G\left(t_{1}, \ldots, t_{i}, t_{i+1}, \ldots, t_{n}\right)=\frac{q^{-1}-q t_{i} / t_{i+1}}{q-q^{-1} t_{i} / t_{i+1}} G\left(t_{1}, \ldots, t_{i+1}, t_{i}, \ldots, t_{n}\right),
$$

where the rational series $\frac{1}{1-x}$ is understood as a series $\sum_{n \geq 0} x^{n}$ and $q$ is a deformation parameter of $U_{q}\left(\widehat{\mathfrak{g l}}_{N}\right)$. Summing the action over the group of permutations we obtain the operator $\overline{\mathrm{Sym}}_{\bar{t}}=$ $\sum_{\sigma \in S_{n}} \pi(\sigma)$ acting as follows:

$$
\overline{\operatorname{Sym}}_{\bar{t}} G(\bar{t})=\frac{1}{n !} \sum_{\sigma \in S_{n}} \prod_{\substack{\ell<\ell^{\prime} \\ \sigma(\ell)>\sigma\left(\ell^{\prime}\right)}} \frac{q^{-1}-q t_{\sigma\left(\ell^{\prime}\right)} / t_{\sigma(\ell)}}{q-q^{-1} t_{\sigma\left(\ell^{\prime}\right)} / t_{\sigma(\ell)}} G\left({ }^{\sigma} t\right)
$$

The product is taken over all pairs $\left(\ell, \ell^{\prime}\right)$, such that conditions $\ell<\ell^{\prime}$ and $\sigma(\ell)>\sigma\left(\ell^{\prime}\right)$ are satisfied simultaneously.

We call operator $\overline{\mathrm{Sym}}_{t}$ a q-symmetrization. One can check that the operation given by (2.2) is a projector

$$
\overline{\operatorname{Sym}}_{\bar{t}} \overline{\operatorname{Sym}}_{\bar{t}}(\cdot)=\overline{\operatorname{Sym}}_{\bar{t}}(\cdot) .
$$

Fix any positive integer $N>1$. Let $\bar{l}=\left\{l_{1}, \ldots, l_{N-1}\right\}$ and $\bar{r}=\left\{r_{1}, \ldots, r_{N-1}\right\}$ be the sets of non-negative integers satisfying a set of inequalities

$$
l_{a} \leq r_{a}, \quad a=1, \ldots, N-1 .
$$

Denote by $[\bar{l}, \bar{r}]$ a set of segments which contain positive integers $\left\{l_{a}+1, l_{a}+2, \ldots, r_{a}-1, r_{a}\right\}$ including $r_{a}$ and excluding $l_{a}$. The length of each segment is equal to $r_{a}-l_{a}$.

For a given set $[\bar{l}, \bar{r}]$ of segments we denote by $\bar{t}_{[\bar{l}, \bar{r}]}$ the sets of variables

$$
\bar{t}_{[\bar{l}, \bar{r}]}=\left\{t_{l_{1}+1}^{1}, \ldots, t_{r_{1}}^{1} ; t_{l_{2}+1}^{2}, \ldots, t_{r_{2}}^{2} ; \ldots ; t_{l_{N-1}+1}^{N-1}, \ldots, t_{r_{N-1}}^{N-1}\right\} .
$$

For any $a=1, \ldots, N-1$ we denote the sets of variables corresponding to the segments $\left[l_{a}, r_{a}\right]=$ $\left\{l_{a}+1, l_{a}+2, \ldots, r_{a}\right\}$ as $\bar{t}_{\left[l_{a}, r_{a}\right]}^{a}=\left\{t_{l_{a}+1}^{a}, \ldots, t_{r_{a}}^{a}\right\}$. All the variables in $\bar{t}_{\left[l_{a}, r_{a}\right]}^{a}$ have the type $a$. For the segments $\left[l_{a}, r_{a}\right]=\left[0, n_{a}\right]$ we use the shorten notations $\bar{t}_{[\overline{0}, \bar{n}]} \equiv \bar{t}_{[\bar{n}]}$ and $\bar{t}_{\left[0, n_{a}\right]}^{a} \equiv \bar{t}_{\left[n_{a}\right]}^{a}$. We also name for a short collections $[\bar{l}, \bar{r}]$ of segments as a segment.

Denote by $S_{\bar{l}, \bar{r}}=S_{l_{1}, r_{1}} \times \cdots \times S_{l_{N-1}, r_{N-1}}$ a direct product of the groups $S_{l_{a}, r_{a}}$ permuting integers $l_{a}+1, \ldots, r_{a}$. Let $G\left(\bar{t}_{[\bar{l}, \bar{r}]}\right)$ be a series depending on the ratios $t_{i}^{a} / t_{j}^{b}$ for $a<b$ and $t_{i}^{a} / t_{j}^{a}$ for $i<j$. The $q$-symmetrization over the whole set of variables $\bar{t}_{[\bar{l}, \bar{r}]}$ of the series $G\left(\bar{t}_{[\bar{l}, \bar{r}]}\right)$ is defined by the formula

$$
\overline{\operatorname{Sym}}_{\bar{t}_{[\bar{l}, \bar{r}]}} G\left(\bar{t}_{[\bar{l}, \bar{r}]}\right)=\sum_{\sigma \in S_{\bar{l}, \bar{r}}} \prod_{1 \leq a \leq N-1} \frac{1}{\left(r_{a}-l_{a}\right) !} \prod_{\substack{\ell<\ell^{\prime} \\ \sigma^{a}(\ell)>\sigma^{a}\left(\ell^{\prime}\right)}} \frac{q^{-1}-q t_{\sigma^{a}\left(\ell^{\prime}\right)}^{a} / t_{\sigma^{a}(\ell)}^{a}}{q-q^{-1} t_{\sigma^{a}\left(\ell^{\prime}\right)}^{a} / t_{\sigma^{a}(\ell)}^{a}} G\left({ }^{\sigma} \bar{t}_{[\bar{l}, \bar{r}]}\right),
$$

where the set ${ }^{\sigma} \bar{t}_{[\bar{l}, \bar{r}]}$ is defined as

$$
{ }^{\sigma} \bar{t}_{[\bar{l}, \bar{r}]}=\left\{t_{\sigma^{1}\left(l_{1}+1\right)}^{1}, \ldots, t_{\sigma^{1}\left(r_{1}\right)}^{1} ; t_{\sigma^{2}\left(l_{2}+1\right)}^{2}, \ldots, t_{\sigma^{2}\left(r_{2}\right)}^{2} ; \ldots ; t_{\sigma^{N-1}\left(l_{N-1}+1\right)}^{N-1}, \ldots, t_{\sigma^{N-1}\left(r_{N-1}\right)}^{N-1}\right\} .
$$

We say that the series $G\left(\bar{t}_{[\bar{l}, \bar{r}]}\right)$ is q-symmetric, if it is invariant under the action $\pi$ of each group $S_{l_{a}, r_{a}}$ with respect to the permutations of the variables $t_{l_{a}+1}^{a}, \ldots, t_{r_{a}}$ for $a=1, \ldots, N-1$ :

$$
\overline{\operatorname{Sym}}_{\bar{t}_{[\bar{l}, \bar{r}]}} G\left(\bar{t}_{[\bar{l}, \bar{r}]}\right)=G\left(\bar{t}_{[\bar{l}, \bar{r}]}\right) .
$$


Due to $(2.3)$ the $q$-symmetrization $G\left(\bar{t}_{[\bar{l}, \bar{r}]}\right)=\overline{\operatorname{Sym}}_{\bar{t}_{[\bar{l}, \bar{r}]}} Q\left(\bar{t}_{[\bar{l}, \bar{r}]}\right)$ of any series $Q\left(\bar{t}_{[\bar{l}, \bar{r}]}\right)$ is a $q$-symmetric series.

The rational function

$$
\prod_{a=1}^{N-1} \prod_{i<j} \frac{1-t_{i}^{a} / t_{j}^{a}}{q-q^{-1} t_{i}^{a} / t_{j}^{a}}
$$

which is understood as a series with respect to $t_{i}^{a} / t_{j}^{a}$ is an example of a $q$-symmetric series.

\subsection{Generating series}

Let $u_{i}, i=1, \ldots, N-1$ be formal parameters. We denote the set of these parameters as $\bar{u}=\left\{u_{1}, \ldots, u_{N-1}\right\}$. Define a generating series

$$
\mathcal{A}(\bar{u})=1+\sum_{\substack{n_{1}, \ldots, n_{N-1} \geq 0 \\ \bar{n} \neq \overline{0}}} \mathcal{A}\left(\bar{t}_{\bar{n}]}\right) u_{1}^{n_{1}} u_{2}^{n_{2}} \cdots u_{N-1}^{n_{N-1}},
$$

where the coefficients $\mathcal{A}\left(\bar{t}_{[\bar{n}]}\right)$ are arbitrary $q$-symmetric series $\left(\overline{\operatorname{Sym}}_{\bar{t}_{[\bar{n}]}}\left(\mathcal{A}\left(\bar{t}_{[\bar{n}]}\right)\right)=\mathcal{A}\left(\bar{t}_{[\bar{n}]}\right)\right)$ of the formal variables $\bar{t}_{\bar{n}}$ numbered by the multi-index $\bar{n}=\left\{n_{1}, \ldots, n_{N-1}\right\}$ :

$$
\bar{t}_{[\bar{n}]}=\left\{t_{1}^{1}, \ldots, t_{n_{1}}^{1} ; t_{1}^{2}, \ldots, t_{n_{2}}^{2} ; \ldots ; t_{1}^{N-2}, \ldots, t_{n_{N-2}-2}^{N-t_{1}^{N-1}}, \ldots, t_{n_{N-1}-1}^{N-1} .\right.
$$

We call generating series of this type q-symmetric generating series. Note that the multi-index $\bar{n}$ of the coefficients of a $q$-symmetric generating series is uniquely defined by the set of formal variables $\bar{t}_{[\bar{n}]}$, thus the coefficients $\mathcal{A}\left(\bar{t}_{[\bar{n}]}\right)$ are used without any additional index. However once it will be convenient for us to use instead the notation $\mathcal{A}_{\bar{n}} \equiv \mathcal{A}\left(\bar{t}_{\bar{n}]}\right)$ (see proof of Proposition 5).

For two generating series $\mathcal{A}(\bar{u})$ and $\mathcal{B}(\bar{u})$ we define $\star$-product as a generating series

$$
\mathcal{C}(\bar{u})=\mathcal{A}(\bar{u}) \star \mathcal{B}(\bar{u})=\sum_{n_{1}, \ldots, n_{N-1} \geq 0} \mathcal{C}\left(\bar{t}_{[\bar{n}]}\right) u_{1}^{n_{1}} u_{2}^{n_{2}} \cdots u_{N-1}^{n_{N-1}}
$$

with coefficients

$$
\mathcal{C}\left(\bar{t}_{[\bar{n}]}\right)=\sum_{0 \leq s_{N-1} \leq n_{N-1}} \cdots \sum_{0 \leq s_{1} \leq n_{1}} \overline{\operatorname{Sym}}_{\bar{t}_{[\bar{n}]}}\left(Z_{\bar{s}}\left(\bar{t}_{[\bar{n}]}\right) \mathcal{A}\left(\bar{t}_{[\bar{s}, \bar{n}]}\right) \cdot \mathcal{B}\left(\bar{t}_{[\overline{0}, \bar{s}]}\right)\right),
$$

where

$$
\mathcal{A}\left(\bar{t}_{[\bar{s}, \bar{n}]}\right)=\mathcal{A}\left(t_{s_{1}+1}^{1}, \ldots, t_{n_{1}}^{1} ; t_{s_{2}+1}^{2}, \ldots, t_{n_{2}}^{2} ; \ldots ; t_{s_{N-1}+1}^{N-1}, \ldots, t_{n_{N-1}}^{N-1}\right)
$$

and

$$
Z_{\bar{s}}\left(\bar{t}_{[\bar{n}]}\right)=\prod_{a=1}^{N-2} \prod_{\substack{s_{a}<\ell \leq n_{a} \\ 0<\ell^{\prime} \leq s_{a+1}}} \frac{q-q^{-1} t_{\ell}^{a} / t_{\ell^{\prime}}^{a+1}}{1-t_{\ell}^{a} / t_{\ell^{\prime}}^{a+1}} .
$$

Proposition 1. The «-product is associative, namely, for three arbitrary q-symmetric series $\mathcal{A}(\bar{u}), \mathcal{B}(\bar{u})$ and $\mathcal{C}(\bar{u})$ of the form $(2.10)$

$$
(\mathcal{A}(\bar{u}) \star \mathcal{B}(\bar{u})) \star \mathcal{C}(\bar{u})=\mathcal{A}(\bar{u}) \star(\mathcal{B}(\bar{u}) \star \mathcal{C}(\bar{u})) .
$$


Proof. We check an equality (2.16) first in the simplest case of the generating series depending on one generating parameter $u$. Equating the coefficients at the $n$-th power of this parameter we obtain from (2.16) an equality

$$
\begin{aligned}
\overline{\operatorname{Sym}}_{\bar{t}} & \left(\sum_{n \geq m \geq 0} \sum_{n \geq s \geq m} \mathcal{A}\left(t_{s+1}, \ldots, t_{n}\right) \cdot \mathcal{B}\left(t_{m+1}, \ldots, t_{s}\right) \cdot \mathcal{C}\left(t_{1}, \ldots, t_{m}\right)\right) \\
& =\overline{\operatorname{Sym}}_{\bar{t}}\left(\sum_{n \geq s^{\prime} \geq 0} \sum_{s^{\prime} \geq m^{\prime} \geq 0} \mathcal{A}\left(t_{s^{\prime}+1}, \ldots, t_{n}\right) \cdot \mathcal{B}\left(t_{m^{\prime}+1}, \ldots, t_{s^{\prime}}\right) \cdot \mathcal{C}\left(t_{1}, \ldots, t_{m^{\prime}}\right)\right),
\end{aligned}
$$

where the property (2.8) of the $q$-symmetric generating series was used and $\bar{t}$ is a set $\left\{t_{1}, \ldots, t_{n}\right\}$. An equality (2.17) is an obvious identity if one replaces the ordering of the summations. It is clear that in the general case the arguments remain the same and the appearing of the series (2.15) does not change these arguments.

Proposition 2. For any generating series $\mathcal{A}(\bar{u})$ there exist an unique q-symmetric series $\mathcal{B}(\bar{u})$ such that

$$
\mathcal{B}(\bar{u}) \star \mathcal{A}(\bar{u})=\mathcal{A}(\bar{u}) \star \mathcal{B}(\bar{u})=1 .
$$

Proof. Since $\mathcal{A}(\bar{u})$ has the form of a Taylor series with the free term equal to 1 , we can always reconstruct uniquely the inverse series solving recursively the equations for the coefficients of the series $\mathcal{B}(\bar{u})$. By the construction the coefficients of this series will be also $q$-symmetric.

\section{Universal nested Bethe vectors for $U_{q}\left(\widehat{\mathfrak{g l}}_{N}\right)$}

Quantum affine algebras in the current realization [2] provide examples of the $q$-symmetric generating series. We will construct these generating series for the quantum affine algebra $U_{q}\left(\widehat{\mathfrak{g l}}_{N}\right)$ and show that $\star$-products of these generating series provide hierarchical relations for NBA. We now recall the current realization of the algebra $U_{q}\left(\widehat{\mathfrak{g l}}_{N}\right)$.

The quantum affine algebra $U_{q}\left(\widehat{\mathfrak{g l}}_{N}\right)$ is generated by the modes of the currents

$$
E_{i}(z)=\sum_{n \in \mathbb{Z}} E_{i}[n] z^{-n}, \quad F_{i}(z)=\sum_{n \in \mathbb{Z}} F_{i}[n] z^{-n}, \quad k_{j}^{ \pm}(z)=\sum_{n \geq 0} k_{j}[ \pm n] z^{\mp n}
$$

where $i=1, \ldots, N-1$ and $j=1, \ldots, N$ subject to the commutation relations given in the Appendix A. The generating series $F_{i}(z), E_{i}(z)$ and $k^{ \pm}(z)$ are called total and Cartan currents respectively.

We consider two types of Borel subalgebras of the algebra $U_{q}\left(\widehat{\mathfrak{g l}}_{N}\right)$.

Generators of the standard Borel subalgebras $U_{q}\left(\mathfrak{b}^{ \pm}\right) \subset U_{q}\left(\widehat{\mathfrak{g l}}_{N}\right)$ can be expressed in terms of the modes of the currents (3.1). To do this one has to introduce the composed currents $E_{a, b}(z)$ and $F_{b, a}(z)$ for $a<b-1$ and $1 \leq a<b \leq N$ (see Appendix A for the definition of the currents $\left.F_{b, a}(z)\right)$. The Borel subalgebra $U_{q}\left(\mathfrak{b}^{+}\right)$is generated by the modes of the currents: $E_{i}[m], m>0 ; F_{i}[n], k_{j}^{+}[n], n \geq 0$ and $E_{a, b}[1], a<b-1$. Dual standard Borel subalgebra $U_{q}\left(\mathfrak{b}^{-}\right)$ is generated by the modes of the currents: $F_{i}[m], m<0 ; E_{i}[n], k_{j}^{+}[n], n \leq 0$ and $F_{b, a}[-1]$, $a<b-1$. Here $i=1, \ldots, N-1$ and $j=1, \ldots, N$. The reader can find description of the standard Borel subalgebras in terms of the modes of the $U_{q}\left(\widehat{\mathfrak{g l}}_{3}\right)$ currents in the paper [9]. This decomposition of the algebra $U_{q}\left(\widehat{\mathfrak{g l}}_{N}\right)$ is related to the standard realization of this algebra in terms of pair of the dual L-operators, where generators of the standard Borel subalgebras serve as the modes of the Gauss coordinates of the corresponding L-operators. 
Another type of Borel subalgebras is related to the current realization of $U_{q}\left(\widehat{\mathfrak{g l}}_{N}\right)$ and was introduced in [2]. The Borel subalgebra $U_{F} \subset U_{q}\left(\widehat{\mathfrak{g l}}_{N}\right)$ is generated by the modes $F_{i}[n], k_{j}^{+}[m]$, $i=1, \ldots, N-1, j=1, \ldots, N, n \in \mathbb{Z}$ and $m \geq 0$. The Borel subalgebra $U_{E} \subset U_{q}\left(\widehat{\mathfrak{g l}}_{N}\right)$ is generated by the modes $E_{i}[n], k_{j}^{-}[-m], i=1, \ldots, N-1, j=1, \ldots, N, n \in \mathbb{Z}$ and $m \geq 0$. We also consider a subalgebra $U_{F}^{\prime} \subset U_{F}$, generated by the elements $F_{i}[n], k_{j}^{+}[m], i=1, \ldots, N-1$, $j=1, \ldots, N, n \in \mathbb{Z}$ and $m>0$, and a subalgebra $U_{E}^{\prime} \subset U_{E}$ generated by the elements $E_{i}[n]$, $k_{j}^{-}[-m], i=1, \ldots, N-1, j=1, \ldots, N, n \in \mathbb{Z}$ and $m>0$. We call these subalgebras of $U_{q}\left(\widehat{\mathfrak{g l}}_{N}\right)$ the current Borel subalgebras. Further, we will be interested in the intersections,

$$
U_{f}^{-}=U_{F}^{\prime} \cap U_{q}\left(\mathfrak{b}^{-}\right), \quad U_{F}^{+}=U_{F} \cap U_{q}\left(\mathfrak{b}^{+}\right)
$$

and will describe properties of projections to these intersections.

The current Borel subalgebras are Hopf subalgebras of $U_{q}\left(\widehat{\mathfrak{g l}}_{N}\right)$ with respect to the current Hopf structure for the algebra $U_{q}\left(\widehat{\mathfrak{g l}}_{N}\right)$ defined in [2]:

$$
\begin{aligned}
& \Delta^{(D)}\left(E_{i}(z)\right)=E_{i}(z) \otimes 1+k_{i}^{-}(z)\left(k_{i+1}^{-}(z)\right)^{-1} \otimes E_{i}(z), \\
& \Delta^{(D)}\left(F_{i}(z)\right)=1 \otimes F_{i}(z)+F_{i}(z) \otimes k_{i}^{+}(z)\left(k_{i+1}^{+}(z)\right)^{-1}, \\
& \Delta^{(D)}\left(k_{i}^{ \pm}(z)\right)=k_{i}^{ \pm}(z) \otimes k_{i}^{ \pm}(z) .
\end{aligned}
$$

The quantum affine algebra $U_{q}\left(\widehat{\mathfrak{g l}}_{N}\right)$ with ommited central charge and gradation operator can be identified with the quantum double of its current Borel subalgebra constructed using the comultiplication (3.3).

One may check that the intersections $U_{f}^{-}$and $U_{F}^{+}$are subalgebras. It was proved in [10] that these subalgebras are coideals with respect to Drinfeld coproduct (3.3)

$$
\Delta^{(D)}\left(U_{F}^{+}\right) \subset U_{q}\left(\widehat{\mathfrak{g l}}_{N}\right) \otimes U_{F}^{+}, \quad \Delta^{(D)}\left(U_{f}^{-}\right) \subset U_{f}^{-} \otimes U_{q}\left(\widehat{\mathfrak{g l}}_{N}\right)
$$

and the multiplication $m$ in $U_{q}\left(\widehat{\mathfrak{g l}}_{N}\right)$ induces an isomorphism of vector spaces

$$
m: \quad U_{f}^{-} \otimes U_{F}^{+} \rightarrow U_{F}
$$

According to the general theory presented in [6] we define projection operators $P^{+}: U_{F} \subset$ $U_{q}\left(\widehat{\mathfrak{g l}}_{N}\right) \rightarrow U_{F}^{+}$and $P^{-}: U_{F} \subset U_{q}\left(\widehat{\mathfrak{g l}}_{N}\right) \rightarrow U_{f}^{-}$by the prescriptions

$$
P^{+}\left(f_{-} f_{+}\right)=\varepsilon\left(f_{-}\right) f_{+}, \quad P^{-}\left(f_{-} f_{+}\right)=f_{-} \varepsilon\left(f_{+}\right), \quad \text { for any } f_{-} \in U_{f}^{-}, f_{+} \in U_{F}^{+},
$$

where $\varepsilon$ is the counit map: $\varepsilon: U_{q}\left(\widehat{\mathfrak{g l}}_{N}\right) \rightarrow \mathbb{C}$.

Denote by $\bar{U}_{F}$ an extension of the algebra $U_{F}$ formed by linear combinations of series, given as infinite sums of monomials $a_{i_{1}}\left[n_{1}\right] \cdots a_{i_{k}}\left[n_{k}\right]$ with $n_{1} \leq \cdots \leq n_{k}$, and $n_{1}+\cdots+n_{k}$ fixed, where $a_{i_{l}}\left[n_{l}\right]$ is either $F_{i_{l}}\left[n_{l}\right]$ or $k_{i_{l}}^{+}\left[n_{l}\right]$. It was proved in [6] that

(1) the projections (3.4) can be extended to the algebra $\bar{U}_{F}$;

(2) for any $f \in \bar{U}_{F}$ with $\Delta^{(D)}(f)=\sum_{i} f_{i}^{\prime} \otimes f_{i}^{\prime \prime}$ we have

$$
f=\sum_{i} P^{-}\left(f_{i}^{\prime \prime}\right) \cdot P^{+}\left(f_{i}^{\prime}\right)
$$




\subsection{Generating series for universal Bethe vectors}

It was proved in the papers $[10,11]$ that the projection of the product of the $U_{q}\left(\widehat{\mathfrak{g l}}_{N}\right)$ currents can be identified with universal Bethe vectors (UBV). In this paper we show that the hierarchical relations for UBV can be presented in a compact form using $\star$-product of certain $q$-symmetric generating series. Then the formal inversion of generating series allows to obtain another form of hierarchical relations and to investigate further (see [8]) special properties of UBV when their parameters satisfy the universal Bethe equations appeared in the framework of the analytical Bethe ansatz [1]. In this paper we will demonstrate these properties for the $U_{q}\left(\widehat{\mathfrak{g l}}_{2}\right)$ universal Bethe vectors.

Products of the $U_{q}\left(\widehat{\mathfrak{g l}}_{N}\right)$ currents yield examples of the $q$-symmetric generating series. We consider the generating series of the product of the currents

$$
\mathcal{F}^{N}(\bar{u})=\sum_{n_{1}, \ldots, n_{N-1} \geq 0} \mathcal{F}^{N}\left(\bar{t}_{[\bar{n}]}\right) u_{1}^{n_{1}} u_{2}^{n_{2}} \cdots u_{N-1}^{n_{N-1}}
$$

where each term $\mathcal{F}^{N}\left(\bar{t}_{[\bar{n}]}\right)$ means the following normalized product of the currents

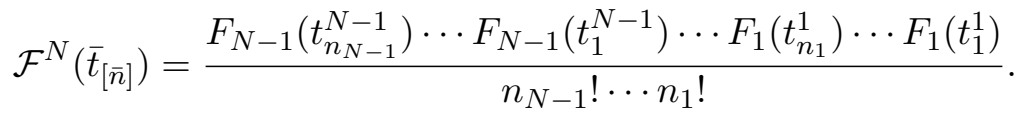

We set $\mathcal{F}_{\overline{0}}^{N} \equiv 1$. More generally, following the convention (2.14), for a segment $[\bar{l}, \bar{r}]$ and the related collection $\bar{t}_{[\bar{l}, \bar{r}]}$ of variables, see $(2.5)$, we set

$$
\mathcal{F}^{N}\left(\bar{t}_{[\bar{l}, \bar{r}]}\right)=\frac{F_{N-1}\left(t_{r_{N-1}}^{N-1}\right) \cdots F_{N-1}\left(t_{l_{N-1}+1}^{N-1}\right) \cdots F_{1}\left(t_{r_{1}}^{1}\right) \cdots F_{1}\left(t_{l_{1}+1}^{1}\right)}{\left(r_{N-1}-l_{N-1}\right) ! \cdots\left(r_{1}-l_{1}\right) !} .
$$

The superscript $N$ in the notation $\mathcal{F}^{N}\left(\bar{t}_{[\bar{n}]}\right)$ of the coefficients of the $q$-symmetric generating series signifies that these coefficients belong to the subalgebra $\bar{U}_{F} \subset U_{q}\left(\widehat{\mathfrak{g l}}_{N}\right)$. Further on we will consider smaller algebras $U_{q}\left(\widehat{\mathfrak{g l}}_{j+1}\right), j=1, \ldots, N-1$ embedded into $U_{q}\left(\widehat{\mathfrak{g l}}_{N}\right)$ in two different way. Embedding $\tau_{j}: U_{q}\left(\widehat{\mathfrak{g l}}_{j+1}\right) \hookrightarrow U_{q}\left(\widehat{\mathfrak{g l}}_{N}\right)$ is defined by removing the currents $F_{j+1}(t), \ldots, F_{N-1}(t)$, $E_{j+1}(t), \ldots, E_{N-1}(t)$ and the Cartan currents $k_{j+2}^{ \pm}(t), \ldots, k_{N}^{ \pm}(t)$. Embedding $\tilde{\tau}_{j}: U_{q}\left(\widehat{\mathfrak{g l}}_{j+1}\right) \hookrightarrow$ $U_{q}\left(\widehat{\mathfrak{g l}}_{N}\right)$ is defined by removing the currents $F_{1}(t), \ldots, F_{N-j-1}(t), E_{1}(t), \ldots, E_{N-j-1}(t)$ and the Cartan currents $k_{1}^{ \pm}(t), \ldots, k_{N-j-1}^{ \pm}(t)$. Note, that the embedding $\tau_{N-1}=\tilde{\tau}_{N-1}$ is an identical map.

Using these embeddings, for any segment $[\bar{l}, \bar{r}]$ such that $l_{i}=r_{i}$ for all $i=N-j+1, N-j+$ $2, \ldots, N-1$ we define a series

$$
\mathcal{F}^{N-j+1}\left(\bar{t}_{[\bar{l}, \bar{r}]}\right)=\frac{F_{N-j}\left(t_{r_{N-j}-j}^{N-j}\right) \cdots F_{N-j}\left(t_{l_{N-j}-1}^{N-1}\right) \cdots F_{1}\left(t_{r_{1}}^{1}\right) \cdots F_{1}\left(t_{l_{1}+1}^{1}\right)}{\left(r_{N-j}-l_{N-j}\right) ! \cdots\left(r_{1}-l_{1}\right) !}
$$

and for any segment $[\bar{l}, \bar{r}]$ such that $l_{i}=r_{i}$ for all $i=1,2, \ldots, j-1$ a series

$$
\tilde{\mathcal{F}}^{N-j+1}\left(\bar{t}_{[\bar{l}, \bar{r}]}\right)=\frac{F_{N-1}\left(t_{r_{N-1}}^{N-1}\right) \cdots F_{N-1}\left(t_{l_{N-1}+1}^{N-1}\right) \cdots F_{j}\left(t_{r_{j}}^{1}\right) \cdots F_{j}\left(t_{l_{j}+1}^{1}\right)}{\left(r_{N-1}-l_{N-1}\right) ! \cdots\left(r_{j}-l_{j}\right) !} .
$$

These series are gathered into $q$-symmetric generating functions $\mathcal{F}^{N-j+1}\left(u_{1}, \ldots, u_{N-j}\right)$ and $\tilde{\mathcal{F}}^{N-j+1}\left(u_{j}, \ldots, u_{N-1}\right)$. Subscripts $a=1, \ldots, N-1$ of the formal parameters $u_{a}$ in the definition of these generating series denote the indices of the simple roots of the algebra $\mathfrak{g l}_{N}$. For example, the notation $\mathcal{F}^{N-1}\left(u_{1}, \ldots, u_{N-2}\right)$ means the generating series which coefficients take values in the subalgebra $U_{q}\left(\widehat{\mathfrak{g l}}_{N-1}\right)$ embedded into $U_{q}\left(\widehat{\mathfrak{g l}}_{N}\right)$ by means of the map $\tau_{N-2}$. On the 
other hand, the notation $\tilde{\mathcal{F}}^{N-1}\left(u_{2}, \ldots, u_{N-1}\right)$ means the generating series taking value in the subalgebra $U_{q}\left(\widehat{\mathfrak{g l}}_{N-1}\right)$ embedded into $U_{q}\left(\widehat{\mathfrak{g l}}_{N}\right)$ by means of the map $\tilde{\tau}_{N-2}$.

Further on we use the special notation for the ordered product of the noncommutative entries. Symbols $\overleftarrow{\prod_{a}} A_{a}$ and $\overrightarrow{\prod_{a}} A_{a}$ will mean ordered products of noncommutative entries $A_{a}$, such that $A_{a}$ is on the right (resp., on the left) from $A_{b}$ for $b>a$ :

$$
\overleftarrow{\prod_{j \geq a \geq i}} A_{a}=A_{j} A_{j-1} \cdots A_{i+1} A_{i}, \quad \prod_{i \leq a \leq j} A_{a}=A_{i} A_{i+1} \cdots A_{j-1} A_{j}
$$

Using these notations we write the $\bar{n}$-th term of the generating series (3.6) as follows

$$
\mathcal{F}^{N}\left(\bar{t}_{[\bar{n}]}\right)=\prod_{N-1 \geq a \geq 1} \frac{1}{n_{a} !}\left(\prod_{n_{a} \geq \ell \geq 1} F_{a}\left(t_{\ell}^{a}\right)\right)
$$

Besides of the generating series of products of the total currents we consider also the generating series of the projections of the products of the currents

$$
P^{ \pm}\left(\mathcal{F}^{N}(\bar{u})\right)=\sum_{n_{1}, \ldots, n_{N-1} \geq 0} P^{ \pm}\left(\mathcal{F}^{N}\left(\bar{t}_{[\bar{n}]}\right)\right) u_{1}^{n_{1}} u_{2}^{n_{2}} \cdots u_{N-1}^{n_{N-1}}
$$

where

$$
P^{ \pm}\left(\mathcal{F}^{N}\left(\bar{t}_{[\bar{n}]}\right)\right)=\frac{P^{ \pm}\left(F_{N-1}\left(t_{n_{N-1}}^{N-1}\right) \cdots F_{N-1}\left(t_{1}^{N-1}\right) \cdots F_{1}\left(t_{n_{1}}^{1}\right) \cdots F_{1}\left(t_{1}^{1}\right)\right)}{n_{N-1} ! \cdots n_{1} !} .
$$

In the same manner we define series

$$
P^{ \pm}\left(\mathcal{F}^{N-j+1}(\bar{u})\right)=P^{ \pm}\left(\mathcal{F}^{N-j+1}\left(u_{1}, \ldots, u_{N-j}\right)\right)
$$

and

$$
P^{ \pm}\left(\tilde{\mathcal{F}}^{N-j+1}(\bar{u})\right)=P^{ \pm}\left(\tilde{\mathcal{F}}^{N-j+1}\left(u_{j}, \ldots, u_{N-1}\right)\right) .
$$

The series $P^{+}\left(\mathcal{F}^{N}(\bar{u})\right)$ is the generating series of all possible universal off-shell Bethe vectors. Our goal is to show that the hierarchical relations of the nested Bethe vectors imply the factorization property of this generating series with respect to the $\star$-product of certain $q$-symmetric generating series. An associativity of this product allows to obtain a new presentation for the universal Bethe vectors.

We call any expression $\sum_{i} f_{-}^{(i)} \cdot f_{+}^{(i)}$, where $f_{-}^{(i)} \in U_{f}^{-}$and $f_{+}^{(i)} \in U_{F}^{+}$(normal) ordered.

Proposition 3. The q-symmetric generating series (3.6) can be written using a $\star$-product in a normal ordered form

$$
\mathcal{F}^{N}(\bar{u})=P^{-}\left(\mathcal{F}^{N}(\bar{u})\right) \star P^{+}\left(\mathcal{F}^{N}(\bar{u})\right),
$$

where the q-symmetric generating series $P^{ \pm}\left(\mathcal{F}^{N}(\bar{u})\right)$ are defined by (3.9).

Proof. Using the property of the projections (3.5) an equality of the series

$$
\mathcal{F}^{N}\left(\bar{t}_{[\bar{n}]}\right)=\sum_{0 \leq s_{N-1} \leq n_{N-1}} \ldots \sum_{0 \leq s_{1} \leq n_{1}} \overline{\operatorname{Sym}}_{\bar{t}_{[\bar{n}]}}\left(Z_{\bar{s}}\left(\bar{t}_{[\bar{n}]}\right) P^{-}\left(\mathcal{F}^{N}\left(\bar{t}_{[\bar{s}, \bar{n}]}\right)\right) \cdot P^{+}\left(\mathcal{F}^{N}\left(\bar{t}_{[\overline{0}, \bar{s}]}\right)\right)\right)
$$

was proved in [11] (see, Proposition 4.1 therein). That proof was based on the comultiplication property (3.3) and the commutation relation between currents. Using a definition of the $\star$-product and considering the coefficients in front of monomial $u_{1}^{n_{1}} \cdots u_{N-1}^{n_{N-1}}$ in the both sides of the equality (3.11) we obtain the formal series equality (3.12). 


\section{Generating series of strings and nested Bethe ansatz}

\subsection{Composed currents and the strings}

For two sets of variables $\left\{t_{1}^{1}, \ldots, t_{k}^{1}\right\}$ and $\left\{t_{1}^{2}, \ldots, t_{k}^{2}\right\}$ we introduce the series

$$
\begin{aligned}
V\left(t_{k}^{2}, \ldots, t_{1}^{2} ; t_{k}^{1}, \ldots, t_{1}^{1}\right) & =\prod_{m=1}^{k} \frac{t_{m}^{1} / t_{m}^{2}}{1-t_{m}^{1} / t_{m}^{2}} \prod_{m^{\prime}=m+1}^{k} \frac{q-q^{-1} t_{m^{\prime}}^{1} / t_{m}^{2}}{1-t_{m^{\prime}}^{1} / t_{m}^{2}} \\
& =\prod_{m=1}^{k} \frac{t_{m}^{1} / t_{m}^{2}}{1-t_{m}^{1} / t_{m}^{2}} \prod_{m^{\prime}=1}^{m-1} \frac{q-q^{-1} t_{m}^{1} / t_{m^{\prime}}^{2}}{1-t_{m}^{1} / t_{m^{\prime}}^{2}} .
\end{aligned}
$$

Fix $j=1, \ldots, N-1$ and a collection of non-negative integers $\bar{s}^{j}=\left\{s_{1}, \ldots, s_{j}\right\}$ satisfying the admissibility condition:

$$
0=s_{0} \leq s_{1} \leq s_{2} \leq \cdots \leq s_{j-1} \leq s_{j} .
$$

We define a series depending on the set of the variables

$$
\bar{t}_{\left[\bar{s}^{j}\right]}=\left\{t_{1}^{1}, \ldots, t_{s_{1}}^{1} ; t_{1}^{2}, \ldots, t_{s_{2}}^{2} ; \ldots ; t_{1}^{j}, \ldots, t_{s_{j}}^{j}\right\}
$$

of the form

$$
X\left(\bar{t}_{\left.\bar{s}^{j}\right]}\right)=\prod_{a=1}^{j-1} V\left(t_{s_{a}}^{a+1}, \ldots, t_{1}^{a+1} ; t_{s_{a}}^{a}, \ldots, t_{1}^{a}\right) .
$$

When $j=1$ we set $X(\cdot)=1$.

Define an ordered normalized product of the composed currents, which we call a string of the type $j:^{1}$

$$
\mathcal{S}^{j+1}\left(\bar{t}_{\left[\bar{s}^{j}\right]}\right)=X\left(\bar{t}_{\left[\bar{s}^{j}\right]}\right) \coprod_{j \geq a \geq 1}\left(\frac{1}{\left(s_{a}-s_{a-1}\right) !} \prod_{s_{a} \geq \ell>s_{a-1}} F_{j+1, a}\left(t_{\ell}^{j}\right)\right)
$$

taking values in the subalgebra $\bar{U}_{F} \subset U_{q}\left(\widehat{\mathfrak{g l}}_{j+1}\right)$ embedded into $U_{q}\left(\widehat{\mathfrak{g l}}_{N}\right)$ by the map $\tau_{j}$. The composed currents $F_{j, i}(t)$ corresponding to non-simple roots of the algebra $\mathfrak{g l}_{N}$ belongs to the completion $\bar{U}_{F}$ and their definition is given in the Appendix A (see (A.3), (A.4), (A.5)).

More generally, let $\bar{m}=\left\{m_{1}, \ldots, m_{N-1}\right\}$ and $\bar{n}=\left\{n_{1}, \ldots, n_{N-1}\right\}$ be a pair of collections of nonnegative integers such that $n_{a}-m_{a}=0$ for $a=j+1, \ldots, N-1$ and $n_{a}-m_{a}=s_{a}$ for any $a=1, \ldots, j$. Then for the set of variables

$$
\bar{t}_{[\bar{m}, \bar{n}]}=\left\{t_{m_{1}+1}^{1}, \ldots, t_{n_{1}}^{1} ; t_{m_{2}}^{2}, \ldots, t_{n_{2}}^{2} ; \ldots ; t_{m_{j}+1}^{j}, \ldots, t_{n_{j}}^{j}\right\},
$$

we set

$$
\begin{aligned}
& X\left(\bar{t}_{[\bar{m}, \bar{n}]}\right)=\prod_{a=1}^{j-1} V\left(t_{m_{a+1}+s_{a}}^{a+1}, \ldots, t_{m_{a+1}+1}^{a+1} ; t_{n_{a}}^{a}, \ldots, t_{m_{a}+1}^{a}\right), \\
& \mathcal{S}^{j+1}\left(\bar{t}_{[\bar{m}, \bar{n}]}\right)=X\left(\bar{t}_{[\bar{m}, \bar{n}]}\right) \overleftarrow{\prod_{j \geq a \geq 1}}\left(\frac{1}{\left(s_{a}-s_{a-1}\right) !} \prod_{s_{a}+m_{j} \geq \ell>s_{a-1}+m_{j}} F_{j+1, a}\left(t_{\ell}^{j}\right)\right) .
\end{aligned}
$$

\footnotetext{
${ }^{1}$ Here the definition of the string differs from those used in $[11,14]$ by the combinatorial factor being a rational function of parameter $t_{i}^{a}$.
} 
We define the $q$-symmetric generating series of the strings of the type $j$ by the formula

$$
\mathcal{S}^{j+1}\left(u_{1}, \ldots, u_{j}\right)=\sum_{s_{j} \geq s_{j-1} \geq \cdots \geq s_{1} \geq 0} \overline{\operatorname{Sym}}_{\bar{t}_{\left[\bar{s}^{j}\right]}}\left(\mathcal{S}^{j+1}\left(\bar{t}_{\left[\bar{s}^{j}\right]}\right)\right) u_{1}^{s_{1}} u_{2}^{s_{2}} \cdots u_{j-1}^{s_{j-1}} u_{j}^{s_{j}} .
$$

Here superscript $j+1$ signifies that this generating series takes values in the subalgebra $U_{q}\left(\widehat{\mathfrak{g l}}_{j+1}\right)$ embedded into $U_{q}\left(\widehat{\mathfrak{g l}}_{N}\right)$ by the map $\tau_{j}$. The subscripts of the parameters $u_{1}, \ldots, u_{j}$ signify that this subalgebra is generated by the $U_{q}\left(\widehat{\mathfrak{g l}}_{N}\right)$ currents corresponding to the simple roots with indices $1, \ldots, j$.

Let $P^{+}\left(\mathcal{F}^{N}\left(u_{1}, \ldots, u_{N-1}\right)\right)$ be the generating series of the universal off-shell Bethe vectors for the algebra $U_{q}\left(\widehat{\mathfrak{g l}}_{N}\right)$ and $P^{+}\left(\mathcal{F}^{N-1}\left(u_{1}, \ldots, u_{N-2}\right)\right)$ be the analogous series for the smaller algebra $U_{q}\left(\widehat{\mathfrak{g l}}_{N-1}\right)$ embedded into $U_{q}\left(\widehat{\mathfrak{g l}}_{N}\right)$ by the map $\tau_{N-2}$. We have the following

Proposition 4. Hierarchical relations between universal weight functions for algebras $U_{q}\left(\widehat{\mathfrak{g l}}_{N}\right)$ and $U_{q}\left(\widehat{\mathfrak{g l}}_{N-1}\right)$ can be written as the following equality on generating series:

$$
P^{+}\left(\mathcal{F}^{N}\left(u_{1}, \ldots, u_{N-1}\right)\right)=P^{+}\left(\mathcal{S}^{N}\left(u_{1}, \ldots, u_{N-1}\right)\right) \star P^{+}\left(\mathcal{F}^{N-1}\left(u_{1}, \ldots, u_{N-2}\right)\right) .
$$

Proof. Taking the coefficients in front of the monomial $u_{1}^{n_{1}} u_{2}^{n_{2}} \cdots u_{N-1}^{n_{N-1}}$ we obtain an equality of the formal series

$$
\begin{aligned}
P^{+}\left(\mathcal{F}^{N}\left(\bar{t}_{[\bar{n}]}\right)\right)= & \sum_{n_{N-1}=s_{N-1} \geq \cdots \geq s_{1} \geq 0} \overline{\operatorname{Sym}}_{\bar{t}_{[\bar{n}]}}\left(Z_{\bar{s}}\left(\bar{t}_{[\bar{n}]}\right)\right. \\
& \left.P^{+}\left(\mathcal{S}^{N}\left(\bar{t}_{[\bar{n}-\bar{s}, \bar{n}]}\right)\right) \cdot P^{+}\left(\mathcal{F}^{N-1}\left(\bar{t}_{[\bar{n}-\bar{s}]}\right)\right)\right) .
\end{aligned}
$$

An equality (4.7) coincides with the statement of the Proposition 4.2 of the paper [11] up to renormalization of the universal weight function by the combinatorial factor.

Corollary 1. Generating series of the $U_{q}\left(\widehat{\mathfrak{g l}}_{N}\right)$ universal weight functions can be written using ordering $\star$-product of the generating series of the strings

$$
\begin{aligned}
P^{+}\left(\mathcal{F}^{N}\left(u_{1}, \ldots, u_{N-1}\right)\right)= & P^{+}\left(\mathcal{S}^{N}\left(u_{1}, \ldots, u_{N-1}\right)\right) \star P^{+}\left(\mathcal{S}^{N-1}\left(u_{1}, \ldots, u_{N-2}\right)\right) \star \cdots \\
& \star P^{+}\left(\mathcal{S}^{3}\left(u_{1}, u_{2}\right)\right) \star P^{+}\left(\mathcal{S}^{2}\left(u_{1}\right)\right) .
\end{aligned}
$$

In (4.8) we assume that the universal Bethe vectors for the algebra $U_{q}\left(\widehat{\mathfrak{g l}}_{1}\right)$ are equal to 1.

Recall that the generating series $P^{+}\left(\mathcal{S}^{j}\left(u_{1}, \ldots, u_{j-1}\right)\right)$ belongs to subalgebra $\bar{U}_{F} \subset U_{q}\left(\widehat{\mathfrak{g l}}_{j}\right)$ embedded into $U_{q}\left(\widehat{\mathfrak{g l}}_{N}\right)$ by the map $\tau_{j-1}$ which removes the currents $F_{a}(t), E_{a}(t)$ and $k_{a+1}^{ \pm}(t)$ with $a=j, j+1, \ldots, N-1$.

\subsection{Hierarchical relations for the negative projections}

One of the results of the papers $[11,14]$ is the hierarchical relation for the positive projections of the product of the currents. Let us recall shortly the main idea of this calculation. In order to calculate the projection

$$
P^{+}\left(F_{N-1}\left(t_{n_{N-1}}^{N-1}\right) \cdots F_{N-1}\left(t_{1}^{N-1}\right) \cdots F_{1}\left(t_{n_{1}}^{1}\right) \cdots F_{1}\left(t_{1}^{1}\right)\right)
$$

we separate all factors $F_{a}\left(t_{\ell}^{a}\right)$ with $a<N-1$ and apply to this product the ordering procedure based on the property (3.12). We obtain under total projection the $q$-symmetrization of terms $x_{i} P^{-}\left(y_{i}\right) P^{+}\left(z_{i}\right)$, where $x_{i}$ are expressed via modes of $F_{N-1}(t)$ and $y_{i}, z_{i}$ via modes of $F_{a}(t)$ with $a<N-1$. Then we used the property of the projection that

$$
P^{+}\left(x_{i} P^{-}\left(y_{i}\right) P^{+}\left(z_{i}\right)\right)=P^{+}\left(x_{i} P^{-}\left(y_{i}\right)\right) \cdot P^{+}\left(z_{i}\right)
$$


and reorder the product of $x_{i}$ and $P^{-}\left(y_{i}\right)$ under positive projection to obtain the string build from the composed currents (cf. equation (4.7)).

We will use an analogous strategy to calculate the negative projection of the same product of the currents

$$
P^{-}\left(F_{N-1}\left(t_{n_{N-1}}^{N-1}\right) \cdots F_{N-1}\left(t_{1}^{N-1}\right) \cdots F_{2}\left(t_{n_{2}}^{2}\right) \cdots F_{2}\left(t_{1}^{2}\right) F_{1}\left(t_{n_{1}}^{1}\right) \cdots F_{1}\left(t_{1}^{1}\right)\right) .
$$

Now we separate all factors $F_{a}\left(t_{\ell}^{a}\right)$ with $a>1$ and apply to this product the ordering rule (3.12). Again, we obtain under total negative projection the $q$-symmetrization of terms $P^{-}\left(x_{i}\right) P^{+}\left(y_{i}\right) z_{i}$, where $z_{i}$ are expressed via modes of $F_{1}(t)$ and $y_{i}, z_{i}$ via modes of $F_{a}(t)$ with $a>1$. Using now the property of the projections

$$
P^{-}\left(P^{-}\left(x_{i}\right) P^{+}\left(y_{i}\right) z_{i}\right)=P^{-}\left(x_{i}\right) \cdot P^{-}\left(P^{+}\left(y_{i}\right) z_{i}\right)
$$

and reordering the product of $P^{+}\left(y_{i}\right)$ and $z_{i}$ under negative projection we obtain the desired hierarchical relations for the negative projection of the currents product.

We will not repeat these calculations since they are analogous to the ones presented in [11], but will formulate the final answer of these hierarchical relations. For two sets of variables $\left\{t_{1}^{1}, \ldots, t_{l}^{1}\right\}$ and $\left\{t_{1}^{2}, \ldots, t_{l}^{2}\right\}$ we introduce the series

$$
\begin{aligned}
\tilde{V}\left(t_{l}^{2}, \ldots, t_{1}^{2} ; t_{l}^{1}, \ldots, t_{1}^{1}\right) & =\prod_{m=1}^{l} \frac{1}{1-t_{m}^{1} / t_{m}^{2}} \prod_{m^{\prime}=m+1}^{l} \frac{q-q^{-1} t_{m^{\prime}}^{1} / t_{m}^{2}}{1-t_{m^{\prime}}^{1} / t_{m}^{2}} \\
& =\prod_{m=1}^{l} \frac{1}{1-t_{m}^{1} / t_{m}^{2}} \prod_{m^{\prime}=1}^{m-1} \frac{q-q^{-1} t_{m}^{1} / t_{m^{\prime}}^{2}}{1-t_{m}^{1} / t_{m^{\prime}}^{2}} .
\end{aligned}
$$

Fix $k=1, \ldots, N-1$ and collection of the non-negative integers $\tilde{s}^{k}=\left\{s_{k}, \ldots, s_{N-1}\right\}$ satisfying the admissibility condition

$$
s_{k} \geq s_{k+1} \geq s_{k+2} \geq \cdots \geq s_{N-1} \geq s_{N}=0 .
$$

We define a series depending on the set of the variables

$$
\bar{t}_{\left[\tilde{s}^{k}\right]}=\left\{t_{1}^{k}, \ldots, t_{s_{k}}^{k} ; t_{1}^{k+1}, \ldots, t_{s_{k+1}}^{k+1} ; \ldots ; t_{1}^{N-1}, \ldots, t_{s_{N-1}}^{N-1}\right\}
$$

of the form

$$
\tilde{X}\left(\bar{t}_{\left[\tilde{s}^{k}\right]}\right)=\prod_{a=k}^{N-2} \tilde{V}\left(t_{s_{a+1}}^{a+1}, \ldots, t_{1}^{a+1} ; t_{s_{a}}^{a}, \ldots, t_{s_{a}-s_{a+1}+1}^{a}\right) .
$$

When $k=N-1$ we set $\tilde{X}(\cdot)=1$.

Define an ordered normalized product of the composed currents, which we call a dual string of the type $N-k$ :

$$
\tilde{\mathcal{S}}^{N-k+1}\left(\bar{t}_{\left[\tilde{s}^{k}\right]}\right)=\tilde{X}\left(\bar{t}_{\left[\tilde{s}^{k}\right]}\right) \coprod_{N \geq a>k}\left(\frac{1}{\left(s_{a-1}-s_{a}\right) !} \coprod_{s_{k}-s_{a} \geq \ell>s_{k}-s_{a-1}} F_{a, k}\left(t_{\ell}^{k}\right)\right)
$$

Note that the notion of the dual string is different from the notion of the inverse string used in [11].

More generally, let $\bar{m}=\left\{m_{1}, \ldots, m_{N-1}\right\}$ and $\bar{n}=\left\{n_{1}, \ldots, n_{N-1}\right\}$ be a pair of collections of nonnegative integers such that $n_{a}-m_{a}=0$ for $a=1, \ldots, k-1$ and $n_{a}-m_{a}=s_{a}$ for any $a=k, \ldots, N-1$. Then for the collection of variables

$$
\bar{t}_{[\bar{m}, \bar{n}]}=\left\{t_{m_{k}+1}^{k}, \ldots, t_{n_{k}}^{k} ; t_{m_{k+1}}^{k+1}, \ldots, t_{n_{k+1}}^{k+1} ; \ldots ; t_{m_{N-1}+1}^{N-1}, \ldots, t_{n_{N-1}}^{N-1}\right\},
$$


we set

$$
\begin{aligned}
& \tilde{X}\left(\bar{t}_{[\bar{m}, \bar{n}]}\right)=\prod_{a=k}^{N-2} \tilde{V}\left(t_{n_{a+1}}^{a+1}, \ldots, t_{m_{a+1}+1}^{a+1} ; t_{n_{a}}^{a}, \ldots, t_{n_{a}-s_{a+1}+1}^{a}\right), \\
& \tilde{\mathcal{S}}^{N-k+1}\left(\bar{t}_{[\bar{m}, \bar{n}]}\right)=\tilde{X}\left(\bar{t}_{[\bar{m}, \bar{n}]}\right) \coprod_{N \geq a>k}\left(\frac{1}{\left(s_{a-1}-s_{a}\right) !} \prod_{n_{k}-s_{a} \geq \ell>n_{k}-s_{a-1}} F_{a, k}\left(t_{\ell}^{k}\right)\right)
\end{aligned}
$$

Doing the calculations described above we obtain the recurrence relations for the negative projections

$$
P^{-}\left(\mathcal{F}^{N}\left(\bar{t}_{[\bar{n}]}\right)\right)=\sum_{n_{1}=s_{1} \geq \cdots \geq s_{N-1} \geq 0} \overline{\operatorname{Sym}}_{\bar{t}_{[\bar{n}]}}\left(Z_{\bar{s}}\left(\bar{t}_{[\bar{n}]}\right) \cdot P^{-}\left(\mathcal{F}^{N-1}\left(\bar{t}_{[\bar{s}, \bar{n}]}\right)\right) \cdot P^{-}\left(\tilde{\mathcal{S}}^{N}\left(\bar{t}_{[\overline{[}]}\right)\right)\right) .
$$

We define the generating series of the dual strings of the type $N-j$ by the formula

$$
\tilde{\mathcal{S}}^{N-k+1}\left(u_{k}, \ldots, u_{N-1}\right)=\sum_{s_{k} \geq s_{k+1} \geq \cdots \geq s_{N-1} \geq 0} \overline{\operatorname{Sym}}_{\bar{t}_{\left[\tilde{s}^{k}\right]}}\left(\tilde{\mathcal{S}}^{N-k+1}\left(\bar{t}_{\left[\tilde{s}^{k}\right]}\right)\right) u_{k}^{s_{k}} u_{k+1}^{s_{k+1}} \cdots u_{N-1}^{s_{N-1}}
$$

taking values in the subalgebra $\bar{U}_{F} \subset U_{q}\left(\widehat{\mathfrak{g l}}_{N-k+1}\right)$ embedded into $U_{q}\left(\widehat{\mathfrak{g l}}_{N}\right)$ by the map $\tilde{\tau}_{N-k}$ which removes the currents $F_{a}(t), E_{a}(t)$ and $k_{a}^{ \pm}(t)$ for $a=1, \ldots, k-1$. The recurrence relations (4.14) can be written as the $\star$-product of the generating series

$$
P^{-}\left(\mathcal{F}^{N}\left(u_{1}, \ldots, u_{N-1}\right)\right)=P^{-}\left(\tilde{\mathcal{F}}^{N-1}\left(u_{2}, \ldots, u_{N-1}\right)\right) \star P^{-}\left(\tilde{\mathcal{S}}^{N}\left(u_{1}, \ldots, u_{N-1}\right)\right) .
$$

Generating series of the negative projections of the product of the currents can be written using ordering $\star$-product of the generating series of the dual strings

$$
\begin{aligned}
P^{-}\left(\mathcal{F}^{N}\left(u_{1}, \ldots, u_{N-1}\right)\right)= & P^{-}\left(\tilde{\mathcal{S}}^{2}\left(u_{N-1}\right)\right) \star P^{-}\left(\tilde{\mathcal{S}}^{3}\left(u_{N-2}, u_{N-1}\right)\right) \star \cdots \\
& \star P^{-}\left(\tilde{\mathcal{S}}^{N-1}\left(u_{2}, \ldots, u_{N-1}\right)\right) \star P^{-}\left(\tilde{\mathcal{S}}^{N}\left(u_{1}, \ldots, u_{N-1}\right)\right) .
\end{aligned}
$$

\subsection{Other type of the hierarchical relations}

A special ordering property of the universal Bethe vectors when their parameters $t_{\ell}^{a}$ satisfy the universal Bethe equations [1] was investigated in [8]. This property leads to the fact that the ordering of the product of the universal transfer matrix and the universal nested Bethe vectors is proportional to the same Bethe vector modulo the terms which belong to some ideal in the algebra if the parameters of this vector satisfy the universal Bethe equations. We will demonstrate this property for the $U_{q}\left(\widehat{\mathfrak{g l}}_{2}\right)$ universal Bethe vectors in the Section 4.7.

A cornerstone of this ordering property lies in a new hierarchical relations for the universal Bethe vectors, which can be proved using the technique of the generating series. Here we give the detailed proof of the relation which particular form was used in the paper [8].

Using normal ordering relation (3.11) and (4.15) we may write the generating series of the product of the currents in the form

$$
\begin{aligned}
& \mathcal{F}^{N}\left(u_{1}, \ldots, u_{N-1}\right)=P^{-}\left(\mathcal{F}^{N}\left(u_{1}, \ldots, u_{N-1}\right)\right) \star P^{+}\left(\mathcal{F}^{N}\left(u_{1}, \ldots, u_{N-1}\right)\right) \\
& \quad=P^{-}\left(\tilde{\mathcal{F}}^{N-1}\left(u_{2}, \ldots, u_{N-1}\right)\right) \star P^{-}\left(\tilde{\mathcal{S}}^{N}\left(u_{1}, \ldots, u_{N-1}\right)\right) \star P^{+}\left(\mathcal{F}^{N}\left(u_{1}, \ldots, u_{N-1}\right)\right) .
\end{aligned}
$$

On the other hand these generating series may be presented as the factorized product

$$
\mathcal{F}^{N}\left(u_{1}, \ldots, u_{N-1}\right)=\tilde{\mathcal{F}}^{N-1}\left(u_{2}, \ldots, u_{N-1}\right) \cdot \mathcal{F}^{2}\left(u_{1}\right)=\tilde{\mathcal{F}}^{N-1}\left(u_{2}, \ldots, u_{N-1}\right) \star \mathcal{F}^{2}\left(u_{1}\right) .
$$


Applying the ordering relation (3.11) to the series $\tilde{\mathcal{F}}^{N-1}\left(u_{2}, \ldots, u_{N-1}\right)$ again we obtain an alternative to (4.17) expression for the generating series $\mathcal{F}^{N}\left(u_{1}, \ldots, u_{N-1}\right)$ :

$$
\begin{aligned}
& \mathcal{F}^{N}\left(u_{1}, \ldots, u_{N-1}\right)=\tilde{\mathcal{F}}^{N-1}\left(u_{2}, \ldots, u_{N-1}\right) \star \mathcal{F}^{2}\left(u_{1}\right) \\
& \quad=P^{-}\left(\tilde{\mathcal{F}}^{N-1}\left(u_{2}, \ldots, u_{N-1}\right)\right) \star P^{+}\left(\tilde{\mathcal{F}}^{N-1}\left(u_{2}, \ldots, u_{N-1}\right)\right) \star \mathcal{F}^{2}\left(u_{1}\right) .
\end{aligned}
$$

Equating the right hand sides of (4.17) and (4.19) we obtain the identity

$$
\begin{gathered}
P^{-}\left(\tilde{\mathcal{S}}^{N}\left(u_{1}, \ldots, u_{N-1}\right)\right) \star P^{+}\left(\mathcal{F}^{N}\left(u_{1}, \ldots, u_{N-1}\right)\right) \\
\quad=P^{+}\left(\tilde{\mathcal{F}}^{N-1}\left(u_{2}, \ldots, u_{N-1}\right)\right) \star \mathcal{F}^{2}\left(u_{1}\right)
\end{gathered}
$$

or

$$
\begin{aligned}
P^{+} & \left(\mathcal{F}^{N}\left(u_{1}, \ldots, u_{N-1}\right)\right) \\
& =\left(P^{-}\left(\tilde{\mathcal{S}}^{N}\left(u_{1}, \ldots, u_{N-1}\right)\right)\right)^{-1} \star P^{+}\left(\mathcal{F}^{N-1}\left(u_{2}, \ldots, u_{N-1}\right)\right) \star \mathcal{F}^{2}\left(u_{1}\right) .
\end{aligned}
$$

The identity (4.21) relates the universal off-shell Bethe vectors for the algebra $U_{q}\left(\widehat{\mathfrak{g l}}_{N}\right)$ and for the smaller algebra $U_{q}\left(\widehat{\mathfrak{g l}}_{N-1}\right)$. They can be considered as an universal formulation of the relation used in the pioneer paper [12] for the obtaining the nested Bethe equations. The equality (4.21) between generating series contains many hierarchical relations between UBV. In order to get some particular identities between these UBV one has to invert explicitly the generating series $P^{-}\left(\tilde{\mathcal{S}}^{N}\left(u_{1}, \ldots, u_{N-1}\right)\right)$. This will be done in the next subsection.

\subsection{Inverting generating series of the strings}

Let $\mathcal{E}(\bar{u})$ be the generating series of negative projections of dual strings of the type $N-1$ : $\mathcal{E}(\bar{u})=P^{-}\left(\tilde{\mathcal{S}}^{N}\left(u_{1}, \ldots, u_{N-1}\right)\right)$ and $\mathcal{D}(\bar{u})$ be the inverse series: $\mathcal{D}(\bar{u}) \star \mathcal{E}(\bar{u})=1$. By the construction, see (4.13), the coefficients

$$
\mathcal{E}\left(\bar{t}_{[\bar{m}]}\right)=P^{-}\left(\overline{\operatorname{Sym}}_{\bar{t}_{[\bar{m}]}}\left(\tilde{\mathcal{S}}^{N}\left(\bar{t}_{[\bar{m}]}\right)\right)\right)
$$

of the generating series $\mathcal{E}(\bar{u})$ are nonzero only if the admissibility conditions $m_{1} \geq m_{2} \geq \cdots \geq$ $m_{N-1} \geq 0$ for the set $\{\bar{m}\}=\left\{m_{1}, m_{2}, \ldots, m_{N-1}\right\}$ are satisfied. We have to find coefficients of the generating series $\mathcal{D}(\bar{u})$ such that $\mathcal{D}(\bar{u}) \star \mathcal{E}(\bar{u})=1$. The latter equality is equivalent to the system of equations

$$
\overline{\operatorname{Sym}}_{\bar{t}_{[\bar{n}]}}\left(\sum_{n_{1} \geq m_{1} \geq 0} \ldots \sum_{\substack{n_{N-1} \geq m_{N-1} \geq 0 \\ m_{1} \geq \cdots \geq m_{N-1}}} Z_{\bar{m}}\left(\bar{t}_{[\bar{n}]}\right) \mathcal{D}\left(\bar{t}_{[\bar{m}, \bar{n}]}\right) \cdot \mathcal{E}\left(\bar{t}_{[\bar{m}]}\right)\right)=0 .
$$

for the unknown functional coefficients $\mathcal{D}\left(\bar{t}_{[\bar{k}]}\right)$ for all possible fixed values of $n_{1}, \ldots, n_{N-1}$.

Proposition 5. The coefficients $\mathcal{D}\left(\bar{t}_{[\bar{k}]}\right)$ are nonzero only if admissibility conditions $k_{1} \geq k_{2} \geq$ $\cdots \geq k_{N-1} \geq 0$ are satisfied.

Proof. can be performed recursively by considering first the cases for $n_{1}>0$ and $n_{2}=\cdots=$ $n_{N-1}=0$. For these values of $\bar{n}$ the series $Z_{\bar{m}}\left(\bar{t}_{[\bar{n}]}\right)=1$ and coefficients of the series in (4.23) depend only on the variables of the first type $t_{1}^{1}, \ldots t_{n_{1}}^{1}$. The relation (4.23) takes in this case the form

$$
\overline{\operatorname{Sym}}_{\bar{t}_{[n]}}\left(\sum_{n_{1} \geq m_{1} \geq 0} \mathcal{D}\left(t_{m_{1}+1}^{1}, \ldots, t_{n_{1}}^{1}\right) \cdot \mathcal{E}\left(t_{1}^{1}, \ldots, t_{m_{1}}^{1}\right)\right)=0,
$$




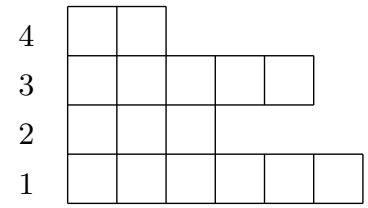

Figure 1. Example of the connected diagram for $n=16, p=4, \chi_{1}=6, \chi_{2}=3, \chi_{3}=5, \chi_{4}=2$.

Its solution can be written in the form

$$
\mathcal{D}\left(\bar{t}_{[n]}\right)=\overline{\operatorname{Sym}}_{\bar{t}_{[n]}}\left(\sum_{p=0}^{n_{1}-1}(-1)^{p+1} \sum_{n_{1}=k_{p+1}>k_{p}>\cdots>k_{1}>k_{0}=0} \coprod_{p+1 \geq r \geq 1} \mathcal{E}\left(t_{k_{r-1}+1}^{1}, \ldots, t_{k_{r}}^{1}\right)\right)
$$

After this we consider the relation (4.23) for arbitrary $n_{1} \geq 0, n_{2}=1$ and $n_{3}=\cdots=n_{N-1}=0$. Avoiding writing the dependence on the ' $t$ ' parameters, that is using notations $\mathcal{E}_{\bar{k}}$ instead of $\mathcal{E}\left(t_{[\bar{k}]}\right)$, the relation (4.23) takes the form

$$
\begin{aligned}
\overline{\operatorname{Sym}}_{\bar{t}_{[\bar{n}]}}\left(\sum_{m_{1}=0}^{n_{1}} \mathcal{D}_{n_{1}-m_{1}, 1, \mathbf{0}} \cdot \mathcal{E}_{m_{1}, 0, \mathbf{0}}\right) \\
\quad+\overline{\operatorname{Sym}}_{\bar{t}_{[\bar{n}]}}\left(\sum_{m_{1}=1}^{n_{1}} Z_{m_{1}, 1, \mathbf{0}} \cdot \mathcal{D}_{n_{1}-m_{1}, 0, \mathbf{0}} \cdot \mathcal{E}_{m_{1}, 1, \mathbf{0}}\right)=0 .
\end{aligned}
$$

The rational series $Z_{m_{1}, 0,0}$ disappears in the first sum of (4.26) by the same reason as in (4.24). Let us consider the relation (4.26) for $n_{1}=0$. The second sum is absent and the first sum contains only one terms $\mathcal{D}_{0,1,0} \cdot \mathcal{E}_{0,0,0}=0$ which is equal to zero. This proves that the coefficient $\mathcal{D}_{0,1,0}=0$ vanishes identically. Now the first sum in the relation (4.26) is terminated at $m_{1}=$ $n_{1}-1$ and this relation allows to find all coefficients $\mathcal{D}_{n_{1}, 1, \mathbf{0}}$ starting from $\mathcal{D}_{1,1, \mathbf{0}}=-Z_{1,1,0} \mathcal{E}_{1,1, \mathbf{0}}$. Considering the relation (4.23) for $n_{1} \geq 0, n_{2}=2$ and $n_{3}=\cdots=n_{N-1}=0$ we prove first that $\mathcal{D}_{0,2,0}=\mathcal{D}_{1,2,0}=0$ and then can find all coefficients $\mathcal{D}_{n_{1}, 2, \mathbf{0}}$ starting from $\mathcal{D}_{2,2, \mathbf{0}}$. It is clear now that the coefficients $\mathcal{D}_{n_{1}, n_{2}, 0}$ are non-zero only if $n_{1} \geq n_{2}$. Continuing we prove the statement of the proposition.

\subsection{Inversion and combinatorics}

To invert explicitly the generating series of the projection of the strings we have to introduce certain combinatorial data. First of all, according to Proposition 5 we fix a sequence of nonnegative integers $n_{1} \geq n_{2} \geq \cdots \geq n_{N-1}$ and the corresponding set of the variables $\bar{t}_{[\bar{n}]}$.

Choose any positive integer $n$ and $p=1, \ldots, n$. A diagram $\chi$ of size $|\chi|=n$ and height $p=\mathrm{h}(\chi)$ is an ordered decomposition of $n$ into a sum of $p$ nonnegative integers,

$$
n=\chi_{1}+\cdots+\chi_{p}
$$

Equivalently, a diagram $\chi$ consists of $p$ rows and the $i$-th row contains $\chi_{i}$ boxes, An example of such diagram is shown in the Fig. 1 . The rows of the diagrams are numbered from the bottom to the top. If $\chi_{i}=0$ for some $i=1, \ldots, p$ then the diagram contains several disconnected pieces. We will call the diagram $\chi$ connected if all $\chi_{i} \neq 0$ for $i=1, \ldots, p$.

A tableaux $\bar{\chi}$ with a given diagram $\chi$ is a filling of all boxes of $\chi$ by the indices $\{1,2, \ldots, N-1\}$ of the positive roots of the algebra $\mathfrak{g l}_{N}$ with the condition of non-increasing from the left to the right along the rows. If an index $a$ is associated to a box of the tableaux, we say that this box has a 'type' $a$. We will call the tableaux associated to the connected diagrams the connected 


\begin{tabular}{|l|l|l|l|l|l|}
\hline 3 & 1 & \multicolumn{5}{|c}{} \\
& 3 & 3 & 2 & 2 & \multicolumn{1}{|c}{1} \\
\hline 1 & 1 & 1 & \multicolumn{3}{|c}{} \\
\hline 2 & 2 & 2 & 1 & 1 & 1 \\
\hline
\end{tabular}

Figure 2. Example of connected tableaux in case of $N=4$ and its weight $\left\{n_{1}, n_{2}, n_{3}\right\}=\{16,8,3\}$.

\begin{tabular}{|c|c|}
\hline 1 & 1 \\
\hline 3 & \\
\hline 3 & 2 \\
\hline
\end{tabular}

\begin{tabular}{|c|c|}
\hline$t_{6}^{1}$ & $t_{5}^{1}$ \\
\hline$t_{2}^{3}, t_{3}^{2}, t_{4}^{1}$ & \\
\hline$t_{1}^{3}, t_{2}^{2}, t_{3}^{1}$ & $t_{1}^{2}, t_{2}^{1}$ \\
\hline
\end{tabular}

Figure 3. Example of tableaux for $N=4$ with associated variables $t_{i}^{a}$.

tableaux. For a given tableaux we define its weight $\bar{n}(\bar{\chi})$ as a set of numbers $\bar{n}=\bar{n}(\bar{\chi})=$ $\left\{n_{1}(\bar{\chi}), \ldots, n_{N-1}(\bar{\chi})\right\}$ such that $n_{a}(\bar{\chi})$ is a number of boxes which have type bigger or equal than $a, a=1, \ldots, N-1$. The size and the height of the tableaux is the size and the height of the corresponding diagram. An example of a connected tableaux is given on the Fig. 2.

Denote by $\bar{\chi}^{i}$ the $i$ th row of the tableaux $\bar{\chi}$. Denote by $c_{a}^{i}=c_{a}^{i}(\bar{\chi})$ the number of type $a$ boxes in the row $\bar{\chi}^{i}$. Set $d_{a}^{i}=d_{a}^{i}(\bar{\chi})=c_{N-1}^{i}+\cdots+c_{a}^{i}$ and $h_{a}^{i}=d_{a}^{1}+\cdots+d_{a}^{i}$ In particular, $d_{1}^{i}(\bar{\chi})$ is the length $\chi_{i}$ of the row $\bar{\chi}^{i}$ and the collection $\bar{d}^{i}=\left\{d_{1}^{i}, \ldots, d_{N-1}^{i}\right\}$ is the character $\bar{n}\left(\bar{\chi}^{i}\right)$ of the row $\bar{\chi}^{i}$ considered as a tableaux by itself. Clearly $d_{a}^{i} \geq d_{b}^{i}$ when $a \leq b$ and

$$
n_{a}=h_{a}^{p}=d_{a}^{1}+\cdots+d_{a}^{p}
$$

for all $a=1, \ldots N-1$. This formula demonstrates in particular that the weight of a tableaux always satisfies the admissibility conditions $n_{1} \geq n_{2} \geq \cdots \geq n_{N-1}$.

To each connected tableaux $\bar{\chi}$ of the weight $\bar{n}=\bar{n}(\bar{\chi})$ we associate a decomposition of the set of the variables $\bar{t}_{[\bar{n}]}$ into the union of $|\chi|$ disjoint subsets, each corresponding to a box of the diagram $\chi$ of tableaux $\bar{\chi}$. To each box of the type $a$ we associate one variable of the type 1 , one variables of the type 2 , etc., one variable of the type $a$, altogether $a$ variables. We will number variables of the each type starting from the most bottom row and the most right box where variable of this type appear for the first time.

Let us give an example of the decomposition and of the ordering for the tableaux shown on the Fig. 3. The most bottom and the right box has the type 1 . We associate to this box one variable $^{2} t_{1}^{1}$ of the same type. Next to the left along the same row box has the type 2 . We associate to this box two variables $t_{2}^{1}$ and $t_{1}^{2}$ of the types 1 and 2 . Last box in the bottom row has type 3 and we associate to this box three variables $t_{3}^{1}, t_{2}^{2}$ and $t_{1}^{3}$. Next box is in the next row and also has the type 3 . To this box we associate also three variables $t_{4}^{1}, t_{3}^{2}$ and $t_{2}^{3}$. Next two boxes in the third row both have the type 1 and we associate to the most right box in this row one variable $t_{5}^{1}$ and to the last box also one variable $t_{6}^{1}$.

In general, for each tableaux $\bar{\chi}$ of the weight $\bar{n}(\bar{\chi})$ and any segment $[\bar{l}, \bar{r}]$, such that $\bar{r}-\bar{l}=\bar{n}$ the set of the variables $\bar{t}_{[\bar{l}, \bar{r}]}$

$$
\bar{t}_{[\bar{l}, \bar{r}]}=\left\{t_{l_{1}+1}^{1}, \ldots, t_{r_{1}}^{1} ; t_{l_{2}+1}^{2}, \ldots, t_{r_{2}}^{2} ; \ldots ; t_{l_{N-1}+1}^{N-1}, \ldots, t_{r_{N-1}}^{N-1}\right\} .
$$

decouples into $\mathrm{h}(\bar{\chi})$ groups of variables

$$
\bar{t}_{\bar{\chi}^{i}}=\left\{t_{l_{1}+h_{1}^{i-1}+1}^{1}, \ldots, t_{l_{1}+h_{1}^{i}}^{1} ; \ldots ; t_{l_{N-1}^{N-1}+h_{N-1}^{i-1}+1}^{N}, \ldots, t_{l_{N-1}+h_{N-1}^{i}}^{N-1}\right\} .
$$

\footnotetext{
${ }^{2}$ Recall that superscript $a$ signifies the 'type' of the variable $t_{i}^{a}$ and subscript $i$ counts the number of the variables of this type.
} 
The variable $t_{k}^{a}$ belongs to the subset $\bar{t}_{\bar{\chi}^{i}}$ if $l_{a}+h_{a}^{i-1}<k \leq l_{a}+h_{a}^{i}$. It is located in the $\left(l_{a}+h_{a}^{i}+1-k\right)$ th box of the row $\bar{\chi}^{i}$ counting boxes in this row from the left edge of the tableaux.

In the same setting we define

$$
Z_{\bar{\chi}}\left(\bar{t}_{[\bar{l}, \bar{r}]}\right)=\prod_{1 \leq i<j \leq \mathrm{h}(\bar{\chi})} Z_{\bar{\chi}^{i}, \bar{\chi}^{j}}\left(\bar{t}_{\bar{\chi}^{i}} ; \bar{t}_{\bar{\chi}^{j}}\right),
$$

where

$$
Z_{\bar{\chi}^{i}, \bar{\chi}^{j}}\left(\bar{t}_{\bar{\chi}^{i}} ; \bar{t}_{\bar{\chi}^{j}}\right)=\prod_{a=1}^{N-2} \prod_{\ell=l_{a}+h_{a}^{j-1}+1}^{l_{a}+h_{a}^{j}} \prod_{\ell^{\prime}=l_{a+1}+h_{a+1}^{i-1}+1}^{l_{a+1}+h_{a+1}^{i}} \frac{q-q^{-1} t_{\ell}^{a} / t_{\ell^{\prime}}^{a+1}}{1-t_{\ell}^{a} / t_{\ell^{\prime}}^{a+1}}
$$

is a rational series defined by the interchanging of the variables of the type $a+1$ from the $i$-th row and variables of the type $a$ from the $j$ th row of the tableaux $\bar{\chi}$.

In our example, the group of variables

$$
\left(t_{2}^{3}, t_{1}^{3} ; t_{3}^{2}, t_{2}^{2}, t_{1}^{2} ; t_{6}^{1}, t_{5}^{1}, t_{4}^{1}, t_{3}^{1}, t_{2}^{1}, t_{1}^{1}\right)
$$

decomposes into three groups

$$
\left(\cdot ; \cdot ; t_{6}^{1}, t_{5}^{1}\right)\left(t_{2}^{3} ; t_{3}^{2} ; t_{4}^{1}\right)\left(t_{1}^{3} ; t_{2}^{2}, t_{1}^{2} ; t_{3}^{1}, t_{2}^{1}, t_{1}^{1}\right) \text {. }
$$

In this example the rational series $Z_{\bar{\chi}}\left(\bar{t}_{[\bar{n}]}\right)$ is equal to

$$
\prod_{\ell=5,6} \frac{q-q^{-1} t_{\ell}^{1} / t_{3}^{2}}{1-t_{\ell}^{1} / t_{3}^{2}} \cdot \prod_{\substack{\ell=4,5,6 \\ \ell^{\prime}=1,2}} \frac{q-q^{-1} t_{\ell}^{1} / t_{\ell^{\prime}}^{2}}{1-t_{\ell}^{1} / t_{\ell^{\prime}}^{2}} \cdot \frac{q-q^{-1} t_{3}^{2} / t_{1}^{3}}{1-t_{3}^{2} / t_{1}^{3}} .
$$

For a given tableaux $\bar{\chi}$ we define the ordered product

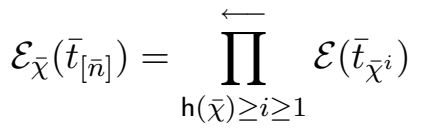

of the negative projections of the strings (4.22). Each factor $\mathcal{E}\left(\bar{t}_{\bar{\chi}^{i}}\right)$ corresponds to the projection of the certain string depending on the set of variables $\bar{t}_{\bar{\chi}^{i}}$ (4.27). Decomposition of the tableaux row into boxes shows the structures of this string. Each box of the type $a$ corresponds to the composed current $F_{a+1,1}$ which depends on the type 1 variable placed in this box. Variables of other types from the same box enter through rational factors. For the tableaux shown on the Fig. 3 this product reads

$$
\mathcal{E}\left(t_{5}^{1}, t_{6}^{1}\right) \cdot \mathcal{E}\left(t_{4}^{1} ; t_{3}^{2} ; t_{2}^{3}\right) \cdot \mathcal{E}\left(t_{1}^{1}, t_{2}^{1}, t_{3}^{1} ; t_{1}^{2}, t_{2}^{2} ; t_{1}^{3}\right)
$$

and

$$
\begin{aligned}
& \mathcal{E}\left(t_{5}^{1}, t_{6}^{1}\right)=P^{-}\left(F_{2,1}\left(t_{6}^{1}\right) F_{2,1}\left(t_{5}^{1}\right)\right), \quad \mathcal{E}\left(t_{4}^{1} ; t_{3}^{2} ; t_{2}^{3}\right)=\frac{1}{1-t_{4}^{1} / t_{3}^{2}} \frac{1}{1-t_{3}^{2} / t_{2}^{3}} P^{-}\left(F_{4,1}\left(t_{4}^{1}\right)\right), \\
& \mathcal{E}\left(t_{1}^{1}, t_{2}^{1}, t_{3}^{1} ; t_{1}^{2}, t_{2}^{2} ; t_{1}^{3}\right)=\frac{1}{1-t_{3}^{1} / t_{2}^{2}} \frac{1}{1-t_{2}^{2} / t_{1}^{3}} \frac{1}{1-t_{2}^{1} / t_{1}^{2}} P^{-}\left(F_{4,1}\left(t_{3}^{1}\right) F_{3,1}\left(t_{2}^{1}\right) F_{2,1}\left(t_{1}^{1}\right)\right) .
\end{aligned}
$$

Proposition 6. The coefficient $\mathcal{D}\left(\bar{t}_{[\bar{n}]}\right)$ of the inverse series $\left(P^{-}\left(\tilde{\mathcal{S}}^{N}\left(u_{1}, \ldots, u_{N-1}\right)\right)\right)^{-1}$ are given

$$
\mathcal{D}\left(\bar{t}_{[\bar{n}]}\right)=\sum_{\bar{\chi}}(-1)^{\mathrm{h}(\bar{\chi})+1} \overline{\operatorname{Sym}}_{\bar{t}_{[\bar{n}]}}\left(Z_{\bar{\chi}}\left(\bar{t}_{[\bar{n}]}\right) \cdot \mathcal{E}_{\bar{\chi}}\left(\bar{t}_{[\bar{n}]}\right)\right)
$$

by the sum over all possible connected tableaux $\bar{\chi}$ such that the weight of tableaux $\bar{n}(\bar{\chi})$ is equal to $\bar{n}$. 
Proof. For arbitrary non-empty set $\bar{n} \neq \overline{0}$ we substitute expression (4.33) into (4.23) to obtain the relation

$$
\begin{aligned}
& \sum_{\substack{\bar{\chi} \\
\bar{n}(\bar{\chi})=\bar{n}}}(-1)^{\mathrm{h}(\bar{\chi})+1} \overline{\operatorname{Sym}}_{\bar{t}_{[\bar{n}]}}\left(Z_{\bar{\chi}}\left(\bar{t}_{[\bar{n}]}\right) \cdot \mathcal{E}_{\bar{\chi}}\left(\bar{t}_{[\bar{n}]}\right)\right) \\
& \quad+\sum_{\substack{\bar{m} \\
\bar{m} \neq \overline{0}}} \sum_{\substack{\left.\bar{n}_{\bar{\chi}} \bar{\chi}^{\prime}\right)=\bar{n}-\bar{m}\\
}}(-1)^{\mathrm{h}\left(\bar{\chi}^{\prime}\right)+1} \overline{\operatorname{Sym}}_{\bar{t}_{[\bar{n}]}}\left(Z_{\bar{m}}\left(\bar{t}_{[\bar{n}]}\right) \cdot Z_{\bar{\chi}^{\prime}}\left(\bar{t}_{[\bar{m}, \bar{n}]}\right) \cdot \mathcal{E}_{\bar{\chi}^{\prime}}\left(\bar{t}_{[\bar{m}, \bar{n}]}\right) \cdot \mathcal{E}\left(\bar{t}_{[\bar{m}]}\right)\right)
\end{aligned}
$$

which has to be equal 0. After this substitution the product of the series $Z_{\bar{\chi}^{\prime}}\left(\bar{t}_{[\bar{m}, \bar{n}]}\right) \cdot \mathcal{E}_{\bar{\chi}^{\prime}}\left(\bar{t}_{[\bar{m}, \bar{n}]}\right)$ should be under $q$-symmetrization over the set of the variables $\bar{t}_{[\bar{m}, \bar{n}]}$. Since the series $Z_{\bar{m}}\left(\bar{t}_{\bar{n}}\right)$ is symmetric with respect to the set of these variables and the series $\mathcal{E}\left(\bar{t}_{\bar{m}}\right)$ does not depend on the variables $\bar{t}_{[\bar{m}, \bar{n}]}$ we may include these series under $q$-symmetrization over the variables $\bar{t}_{[\bar{m}, \bar{n}]}$. Then, since the variables $\bar{t}_{[\bar{m}, \bar{n}]}$ forms a subset of the variables $\bar{t}_{[\bar{n}]}$, the $q$-symmetrization over variables $\bar{t}_{[\bar{m}, \bar{n}]}$ disappear due to the property (2.3).

We will prove the cancellation of the terms in (4.34) in the sums over tableaux of the fixed height. Keep the terms in the summation of the first line of this relation which correspond to the connected tableaux $\bar{\chi}$ such that $n_{a}(\bar{\chi})=n_{a}$ and $\mathrm{h}(\bar{\chi})=p+1$. Keep the terms in the summation of the second line of (4.34) which correspond to all connected tableaux $\bar{\chi}^{\prime}$ such that $n_{a}\left(\bar{\chi}^{\prime}\right)=n_{a}-m_{a}$ and $\mathrm{h}\left(\bar{\chi}^{\prime}\right)=p$ for fixed $p=0, \ldots, n_{1}-1$.

Fix a term in the first sum of (4.34) corresponding to some connected tableaux $\bar{\chi}$ with a weight $\bar{n}$. Consider the first (bottom) line of the tableaux $\bar{\chi}$. Denote by $m_{a}=c_{a}^{1}+\cdots+c_{N-1}^{1}$ the nonnegative integers defined by this row. It is clear that this set of integers satisfies the admissibility condition $m_{1} \geq m_{2} \geq \cdots \geq m_{N-1}$ and $m_{a} \leq n_{a}$. In the second double sum of (4.34) choose the term corresponding to this set $\bar{m}$ and the tableaux $\bar{\chi}^{\prime}$ defined by the following rule. If we glue from the bottom of the tableaux $\bar{\chi}^{\prime}$ the row of boxes such that it has a length $m_{1}$ and the number of boxes of the type $a$ is equal to $m_{a}-m_{a+1}$ then for the obtained tableaux $\tilde{\chi}$ we require $r_{a}^{i}(\tilde{\chi})=r_{a}^{i}(\bar{\chi})$ and $\mathrm{h}(\tilde{\chi})=p+1$ for all possible values $i$ and $a$. We claim that for each fixed tableaux $\bar{\chi}$ there are unique set $\bar{m}$ such that $m_{a} \leq n_{a}$ and there are a single tableaux $\bar{\chi}^{\prime}$ which satisfies above conditions. The tableaux $\tilde{\chi}$ and $\bar{\chi}$ coincide actually. The product of the coefficients $\mathcal{E}_{\bar{\chi}^{\prime}}\left(\bar{t}_{[\bar{m}, \bar{n}]}\right) \cdot \mathcal{E}\left(\bar{t}_{\bar{m}}\right)$ will be equal to $\mathcal{E}_{\bar{\chi}}\left(\bar{t}_{[\bar{n}]}\right)$.

According to the definitions of the series $Z_{\bar{m}}\left(\bar{t}_{\bar{n}}\right)$ (2.15) and $Z_{\bar{\chi}^{\prime}}\left(\bar{t}_{[\bar{m}, \bar{n}]}\right)$ (4.28) their product will be equal to the series $Z_{\bar{\chi}}\left(\bar{t}_{\bar{n}]}\right)$. The term corresponding to the fixed tableaux $\bar{\chi}$ in the first line of (4.34) and the term from the second line given by $\bar{m}$ and $\bar{\chi}^{\prime}$ described above cancel each other since they will enter with different signs: $\mathrm{h}(\bar{\chi})=\mathrm{h}\left(\bar{\chi}^{\prime}\right)+1=p+1$.

For the example of the tableaux shown in the Fig. 3 the sets $\bar{t}_{[\bar{m}]}$ and $\bar{t}_{[\bar{m}, \bar{n}]}$ are

$$
\left\{t_{1}^{3} ; t_{2}^{2}, t_{1}^{2} ; t_{3}^{1}, t_{2}^{1}, t_{1}^{1}\right\} \quad \text { and } \quad\left\{t_{2}^{3} ; t_{3}^{2} ; t_{6}^{1}, t_{5}^{1}, t_{4}^{1}\right\}
$$

respectively. The series $Z_{\bar{m}}\left(\bar{t}_{\bar{n}}\right)$ is

$$
\prod_{\substack{\ell=4,5,6 \\ \ell^{\prime}=1,2}} \frac{q-q^{-1} t_{\ell}^{1} / t_{\ell^{\prime}}^{2}}{1-t_{\ell}^{1} / t_{\ell^{\prime}}^{2}} \cdot \frac{q-q^{-1} t_{3}^{2} / t_{1}^{3}}{1-t_{3}^{2} / t_{1}^{3}} .
$$

The series $Z_{\bar{\chi}^{\prime}}\left(\bar{t}_{[\bar{m}, \bar{n}]}\right)$ is

$$
\prod_{\ell=5,6} \frac{q-q^{-1} t_{\ell}^{1} / t_{3}^{2}}{1-t_{\ell}^{1} / t_{3}^{2}}
$$

The product of (4.35) and (4.36) obviously coincides with (4.31). 


\subsection{Inversion of generating series for $U_{q}\left(\widehat{\mathfrak{g l}}_{2}\right)$}

Quantum affine algebra $U_{q}\left(\widehat{\mathfrak{g l}}_{2}\right)$ in its current realization formed by the modes of the currents ${ }^{3}$ $E(t), F(t)$ and Cartan currents $k_{1}^{ \pm}(t), k_{2}^{ \pm}(t)$.

Let us invert explicitly the generating series $P^{-}(\mathcal{F}(\bar{u}))$ in the simplest case of one generating parameters $\bar{u}=u_{1} \equiv u$ in (3.11) to show what kind of relations can be obtained for the generating series of the $U_{q}\left(\widehat{\mathfrak{g l}}_{2}\right)$ off-shell Bethe vectors $P^{+}(\mathcal{F}(u))$ :

$$
P^{+}(\mathcal{F}(u))=P^{-}(\mathcal{F}(u))^{-1} \star \mathcal{F}(u) .
$$

For any positive $n$ and non-negative $p \leq n$ we define the set of $p+1$ positive integers $\left\{\bar{k}_{p}\right\}=\left\{k_{1}, k_{2}, \ldots, k_{p}, k_{p+1}\right\}$ such that $0=k_{0}<k_{1}<k_{2}<\cdots<k_{p}<k_{p+1}=n$. Using this data we define the ordered products

$$
\mathbf{F}_{\bar{k}_{p}}\left(\bar{t}_{[n]}\right)=\prod_{p+1 \geq m \geq 1} \frac{1}{\left(k_{m}-k_{m-1}\right) !} P^{-}\left(F\left(t_{k_{m}}\right) \cdots F\left(t_{k_{m-1}+1}\right)\right)
$$

It is clear that the inverse generating series $P^{-}(\mathcal{F}(u))$ can be written using $q$-symmetrization of these ordered products $\mathbf{F}_{\bar{k}_{p}}\left(\bar{t}_{[n]}\right)$ as follows

$$
P^{-}(\mathcal{F}(u))^{-1}=1+\sum_{n>0} \overline{\operatorname{Sym}}_{\bar{t}_{[n]}}\left(\sum_{p=0}^{n-1}(-1)^{p+1} \sum_{\left\{\bar{k}_{p}\right\}} \mathbf{F}_{\bar{k}_{p}}\left(\bar{t}_{[n]}\right)\right) u^{n} .
$$

Then using the definition of the $\star$-product we can obtain from (4.37) a special presentation for the $U_{q}\left(\widehat{\mathfrak{g l}}_{2}\right)$ universal weight function

$$
P^{+}\left(\mathcal{F}\left(\bar{t}_{[n]}\right)\right)=\overline{\operatorname{Sym}}_{\bar{t}_{[n]}}\left(\sum_{s=0}^{n} \sum_{p=0}^{n-s-1}(-1)^{p+1} \sum_{\left\{k_{p}\right\}} \mathbf{F}_{\bar{k}_{p}}\left(\bar{t}_{[s, n]}\right) \cdot \mathcal{F}\left(\bar{t}_{[s]}\right)\right),
$$

where summation over the set $\left\{\bar{k}_{p}\right\}$ runs over all possible $k_{i}$ such that $s=k_{0}<k_{1}<k_{2}<$ $\cdots<k_{p}<k_{p+1}=n$. An extreme term in the sum when $s=n$ and the sum over $p$ is absent corresponds to the product of the total currents $F\left(t_{n}\right) \cdots F\left(t_{1}\right)$. Note that an equality (4.39) can be treated as generalization of Ding-Frenkel relation $P^{+}(F(t))=F(t)-P^{-}(F(t))[3]$ when the positive projection is taken from the product of the currents.

\subsection{Universal Bethe ansatz for $U_{q}\left(\widehat{\mathfrak{g l}}_{2}\right)$}

Let $J$ be the left ideal of $U_{q}\left(\mathfrak{b}^{+}\right) \subset U_{q}\left(\widehat{\mathfrak{g l}}_{2}\right)$, generated by all element of the form $U_{q}\left(\mathfrak{b}^{+}\right) \cdot E[n]$, $n>0$. As it was mentioned above the standard Borel subalgebra $U_{q}\left(\mathfrak{b}^{+}\right)$in terms of the currents generators is formed by the modes $F[n], k_{1,2}^{+}[n], n \geq 0$ and $E[m], m>0$. Let us denote subalgebras generated by these modes as $U_{f}^{+}, U_{k}^{+}$and $U_{e}^{+}$, respectively. The multiplication in $U_{q}\left(\mathfrak{b}^{+}\right)$implies an isomorphism of the vectors spaces

$$
U_{f}^{+} \otimes U_{k}^{+} \otimes U_{e}^{+} \rightarrow U_{q}\left(\mathfrak{b}^{+}\right) .
$$

We introduce ordering of the generators in the Borel subalgebra $U_{q}\left(\mathfrak{b}^{+}\right)$

$$
U_{f}^{+} \prec U_{k}^{+} \prec U_{e}^{+}
$$

\footnotetext{
${ }^{3}$ Since algebra $\mathfrak{g l}_{2}$ has only one root we remove index of this simple root in the notation of the currents in case of the current realization of the algebra $U_{q}\left(\widehat{\mathfrak{g l}}_{2}\right)$.
} 
induced by the circular ordering of the Cartan-Weyl generators in the whole algebra $U_{q}\left(\widehat{\mathfrak{g l}}_{2}\right)[6]$. We call any element $w \in U_{q}\left(\mathfrak{b}^{+}\right)$normal ordered and denote it as : $W:$ if it is presented as the linear combination of the elements of the form $W_{1} \cdot W_{2} \cdot W_{3}$, where $W_{1} \in U_{f}^{+}, W_{2} \in U_{k}^{+}, W_{3} \in U_{e}^{+}$. It is convenient to gather the generators of the subalgebras $U_{f}^{+}$and $U_{e}^{+}$into generating series

$$
F^{+}(t)=\sum_{n \geq 0} F[n] t^{-n}, \quad E^{+}(t)=\sum_{n>0} E[n] t^{-n}
$$

which we call the half-currents.

A universal transfer matrix is the following combinations of the Cartan and half-currents

$$
\mathcal{T}(t)=k_{1}^{+}(t)+F^{+}(t) k_{2}^{+}(t) E^{+}(t)+k_{2}^{+}(t) .
$$

Using the commutation relations in the algebra $U_{q}\left(\mathfrak{b}^{+}\right) \subset U_{q}\left(\widehat{\mathfrak{g l}}_{2}\right)$ one may check that these transfer matrices commute for the different values of the spectral parameters

$$
\left[\mathcal{T}(t), \mathcal{T}\left(t^{\prime}\right)\right]=0
$$

and so generates the infinite set of commuting quantities ${ }^{4}$. We are interesting in the ordering relations between universal transfer matrix $\mathcal{T}(t)$ and the universal Bethe vector $P^{+}\left(\mathcal{F}\left(\bar{t}_{[n]}\right)\right)$. Note that the universal transfer matrix is ordered according to the ordering (4.40).

Proposition 7. A formal series identity is valid in $U_{q}\left(\mathfrak{b}^{+}\right)$

$$
: \mathcal{T}(t) \cdot P^{+}\left(\mathcal{F}\left(\bar{t}_{[n]}\right)\right):=P^{+}\left(\mathcal{F}\left(\bar{t}_{[n]}\right)\right) \cdot \tau\left(t ; \bar{t}_{[n]}\right) \quad \bmod J
$$

modulo elements of the left ideal $J$ if the set $\left\{t_{j}\right\}$ of the Bethe parameters satisfies the set of the universal Bethe equations [1], $j=1, \ldots, n$ :

$$
\frac{k_{1}^{+}\left(t_{j}\right)}{k_{2}^{+}\left(t_{j}\right)}=\prod_{m \neq j}^{n} \frac{q t_{j}-q^{-1} t_{m}}{q^{-1} t_{j}-q t_{m}}
$$

and

$$
\tau\left(t ; \bar{t}_{[n]}\right)=k_{1}^{+}(t) \prod_{j=1}^{n} \frac{q^{-1} t-q t_{j}}{t-t_{j}}+k_{2}^{+}(t) \prod_{j=1}^{n} \frac{q t-q^{-1} t_{j}}{t-t_{j}}
$$

is an eigenvalue of the universal transfer matrix.

Proof. Recall that a universal Bethe vector in the considered case coincides with projection of the product of the currents: $P^{+}\left(\mathcal{F}\left(\bar{t}_{[n]}\right)\right)=P^{+}\left(F\left(t_{n}\right) \cdots F\left(t_{1}\right)\right) / n$ ! and can be presented as factorized product of the linear combinations of the half-currents $F^{+}\left(t_{i}\right)[6,9]$. A direct way to prove the statement of the Proposition 7 is to use the commutation relations in $U_{q}\left(\mathfrak{b}^{+}\right)$between half-currents

$$
\begin{aligned}
& {\left[E^{+}(t), F^{+}\left(t^{\prime}\right)\right]=\frac{\left(q-q^{-1}\right) t^{\prime}}{t-t^{\prime}}\left(k_{1}^{+}\left(t^{\prime}\right) k_{2}^{+}\left(t^{\prime}\right)^{-1}-k_{1}^{+}(t) k_{2}^{+}(t)^{-1}\right),} \\
& k_{2}^{+}(t) F^{+}\left(t^{\prime}\right) k_{2}^{+}(t)^{-1}=\frac{q t-q^{-1} t^{\prime}}{t-t^{\prime}} F^{+}\left(t^{\prime}\right)-\frac{\left(q-q^{-1}\right) t^{\prime}}{t-t^{\prime}} F^{+}(t)
\end{aligned}
$$

and similar for $k_{1}^{+}(t) F^{+}\left(t^{\prime}\right) k_{1}^{+}(t)^{-1}$ to present the product $\mathcal{T}(t) \cdot P^{+}\left(\mathcal{F}\left(\bar{t}_{[n]}\right)\right)$ in the normal ordered form. But this way is not easy even in the simplest case of the algebra $U_{q}\left(\widehat{\mathfrak{g l}}_{2}\right)$. It

\footnotetext{
${ }^{4} \mathrm{~A}$ standard way to prove this commutativity is to note that (4.41) is a trace of the fundamental L-operators for $U_{q}\left(\widehat{\mathfrak{g l}}_{2}\right)$ and the commutativity follows from the Yang-Baxter equation for these L-operators.
} 
becomes much more involved in the general case of the algebra $U_{q}\left(\widehat{\mathfrak{g l}}_{N}\right)$. There is a simple way to avoid these difficulties using the relation (4.39).

This relation allows to replace the projection of the product of the currents onto positive Borel subalgebra $U_{q}\left(\mathfrak{b}^{+}\right)$by the linear combination of the terms

$$
\begin{aligned}
P^{+}\left(F\left(t_{n}\right) \cdots F\left(t_{1}\right)\right)= & F\left(t_{n}\right) \cdots F\left(t_{1}\right) \\
& -n \overline{\operatorname{Sym}}_{\bar{t}}\left(P^{-}\left(F\left(t_{n}\right)\right) \cdot F\left(t_{n-1}\right) \cdots F\left(t_{1}\right)\right)+W,
\end{aligned}
$$

where $W$ are the terms which have on the left the product of at least two negative projections

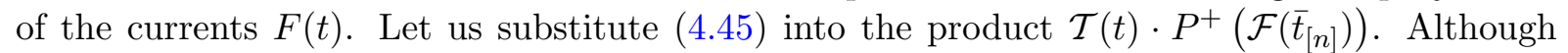
left hand side of (4.45) belongs to the positive Borel subalgebra, each term in the right hand side of the equality (4.45) does not belong to $U_{q}\left(\mathfrak{b}^{+}\right)$. The product of the universal transfer matrix with these terms will produce the terms such that some of them belong to $U_{q}\left(\mathfrak{b}^{+}\right)$and other does not belong to $U_{q}\left(\mathfrak{b}^{+}\right)$. The latter terms which after ordering do not belong to $U_{q}\left(\mathfrak{b}^{+}\right)$ can be omitted since we are interesting only in the terms which belong to the positive Borel subalgebras. In particular, one may check that : $\mathcal{T}(t) \cdot W: \notin U_{q}\left(\mathfrak{b}^{+}\right)$, where $W$ are the terms in (4.45) not showing explicitly (see [8] for details). Using the commutation relations of the half-currents $E^{+}(t)$ with total currents $F\left(t^{\prime}\right)$

$$
\left[E^{+}(t), F\left(t^{\prime}\right)\right]=\frac{\left(q-q^{-1}\right) t^{\prime}}{t-t^{\prime}}\left(k_{1}^{+}\left(t^{\prime}\right) k_{2}^{+}\left(t^{\prime}\right)^{-1}-k_{1}^{-}\left(t^{\prime}\right) k_{2}^{-}\left(t^{\prime}\right)^{-1}\right),
$$

the commutation relations of the Cartan currents with total currents $F\left(t^{\prime}\right)$

$$
k_{2}^{+}(t) F\left(t^{\prime}\right) k_{2}^{+}(t)^{-1}=\frac{q t-q^{-1} t^{\prime}}{t-t^{\prime}} F^{+}\left(t^{\prime}\right), \quad k_{1}^{+}(t) F\left(t^{\prime}\right) k_{1}^{+}(t)^{-1}=\frac{q^{-1} t-q t^{\prime}}{t-t^{\prime}} F^{+}\left(t^{\prime}\right)
$$

and the commutation relations of $E^{+}(t), k_{1}^{+}(t), k_{2}^{+}(t)$ with $P^{-}\left(F\left(t^{\prime}\right)\right)$ we may check that the only terms which belong to the positive Borel subalgebra $U_{q}\left(\mathfrak{b}^{+}\right)$and do not belong to the left ideal $J$ in the normal ordered product $: \mathcal{T}(t) \cdot P^{+}\left(F\left(t_{n}\right) \cdots F\left(t_{1}\right)\right)$ : are

$$
\begin{aligned}
: \mathcal{T}(t) & \cdot P^{+}\left(F\left(t_{n}\right) \cdots F\left(t_{1}\right)\right): \\
= & P^{+}\left(F\left(t_{n}\right) \cdots F\left(t_{1}\right)\right)\left(\prod_{i=1}^{n} \frac{q^{-1} t-q t_{i}}{t-t_{i}} k_{1}^{+}(t)+\prod_{i=1}^{n} \frac{q t-q^{-1} t_{i}}{t-t_{i}} k_{2}^{+}(t)\right) \\
& +n \overline{\operatorname{Sym}}_{\bar{t}}\left(P^{+}\left(F^{+}(t) k_{2}^{+}(t) F\left(t_{n}\right) \cdots F\left(t_{2}\right)\right) \frac{\left(q-q^{-1}\right) t_{1}}{t-t_{1}} k_{1}^{+}\left(t_{1}\right) k_{2}^{+}\left(t_{1}\right)^{-1}\right) \\
& -n \overline{\operatorname{Sym}}_{\bar{t}}\left(P^{+}\left(F^{+}(t) k_{2}^{+}(t) F\left(t_{n-1}\right) \cdots F\left(t_{1}\right)\right) \frac{\left(q-q^{-1}\right) t_{n}}{t-t_{n}}\right) .
\end{aligned}
$$

In order to prove the statement of the Proposition 7 we have to cancel the last two terms in (4.46). This can be done using the properties of the $q$-symmetrization that for any formal series $G\left(t_{1}, \ldots, t_{n}\right)$ on $n$ formal variables $t_{i}$ we have

$$
n \overline{\operatorname{Sym}}_{\bar{t}} G\left(t_{1}, \ldots, t_{n}\right)=\sum_{m=1}^{n} \prod_{j=m+1}^{n} \frac{q^{-1} t_{m}-q t_{j}}{q t_{m}-q^{-1} t_{j}} \overline{\operatorname{Sym}}_{\bar{t} \backslash t_{m}} G\left(t_{1}, \ldots, t_{m-1}, t_{m+1}, \ldots, t_{n}, t_{m}\right)
$$

and

$$
n \overline{\operatorname{Sym}}_{\bar{t}} G\left(t_{1}, \ldots, t_{n}\right)=\sum_{m=1}^{n} \prod_{j=1}^{m-1} \frac{q^{-1} t_{j}-q t_{m}}{q t_{j}-q^{-1} t_{m}} \overline{\operatorname{Sym}}_{\bar{t} t_{m}} G\left(t_{m}, t_{1}, \ldots, t_{m-1}, t_{m+1}, \ldots, t_{n}\right),
$$

where $q$-symmetrization in the right hand sides of this formal series identities runs over $(n-1)$ variables $\bar{t} \backslash t_{m}=\left\{t_{1}, \ldots, t_{m-1}, t_{m+1}, \ldots, t_{m}\right\}$. Using these relations we conclude that last two terms in (4.46) cancel each other provided the relation (4.43) is satisfied. 


\section{A Current realization of $U_{q}\left(\widehat{\mathfrak{g l}}_{N}\right)$}

The commutation relations for the algebra $U_{q}\left(\widehat{\mathfrak{g l}}_{N}\right)$ in the current realization are given by the following set of the relations

$$
\begin{aligned}
& \left(q^{-1} z-q w\right) E_{i}(z) E_{i}(w)=E_{i}(w) E_{i}(z)\left(q z-q^{-1} w\right), \\
& (z-w) E_{i}(z) E_{i+1}(w)=E_{i+1}(w) E_{i}(z)\left(q^{-1} z-q w\right), \\
& k_{i}^{ \pm}(z) E_{i}(w)\left(k_{i}^{ \pm}(z)\right)^{-1}=\frac{z-w}{q^{-1} z-q w} E_{i}(w), \\
& k_{i+1}^{ \pm}(z) E_{i}(w)\left(k_{i+1}^{ \pm}(z)\right)^{-1}=\frac{z-w}{q z-q^{-1} w} E_{i}(w), \\
& k_{i}^{ \pm}(z) E_{j}(w)\left(k_{i}^{ \pm}(z)\right)^{-1}=E_{j}(w), \quad \text { if } \quad i \neq j, j+1, \\
& \left(q z-q^{-1} w\right) F_{i}(z) F_{i}(w)=F_{i}(w) F_{i}(z)\left(q^{-1} z-q w\right), \\
& \left(q^{-1} z-q w\right) F_{i}(z) F_{i+1}(w)=F_{i+1}(w) F_{i}(z)(z-w), \\
& k_{i}^{ \pm}(z) F_{i}(w)\left(k_{i}^{ \pm}(z)\right)^{-1}=\frac{q^{-1} z-q w}{z-w} F_{i}(w), \\
& k_{i+1}^{ \pm}(z) F_{i}(w)\left(k_{i+1}^{ \pm}(z)\right)^{-1}=\frac{q z-q^{-1} w}{z-w} F_{i}(w), \\
& k_{i}^{ \pm}(z) F_{j}(w)\left(k_{i}^{ \pm}(z)\right)^{-1}=F_{j}(w), \quad \text { if } \quad i \neq j, j+1, \\
& {\left[E_{i}(z), F_{j}(w)\right]=\delta_{i, j} \delta(z / w)\left(q-q^{-1}\right)\left(k_{i}^{+}(z) / k_{i+1}^{+}(z)-k_{i}^{-}(w) / k_{i+1}^{-}(w)\right)}
\end{aligned}
$$

and the Serre relations for the currents $E_{i}(z)$ and $F_{i}(z)$

$$
\begin{aligned}
& \operatorname{Sym}_{z_{1}, z_{2}}\left(E_{i}\left(z_{1}\right) E_{i}\left(z_{2}\right) E_{i \pm 1}(w)-\left(q+q^{-1}\right) E_{i}\left(z_{1}\right) E_{i \pm 1}(w) E_{i}\left(z_{2}\right)\right. \\
& \left.\quad+E_{i \pm 1}(w) E_{i}\left(z_{1}\right) E_{i}\left(z_{2}\right)\right)=0 \\
& \operatorname{Sym}_{z_{1}, z_{2}}\left(F_{i}\left(z_{1}\right) F_{i}\left(z_{2}\right) F_{i \pm 1}(w)-\left(q+q^{-1}\right) F_{i}\left(z_{1}\right) F_{i \pm 1}(w) F_{i}\left(z_{2}\right)\right. \\
& \left.\quad+F_{i \pm 1}(w) F_{i}\left(z_{1}\right) F_{i}\left(z_{2}\right)\right)=0 .
\end{aligned}
$$

Formulae (A.1) and (A.2) should be considered as formal series identities describing the infinite set of the relations between modes of the currents. The symbol $\delta(z)$ entering these relations is a formal series $\sum_{n \in \mathbb{Z}} z^{n}$.

Following $[4,9]$, we introduce composed currents $F_{j, i}(t)$ for $i<j$. The composed currents for nontwisted quantum affine algebras were defined in [4]. According to this paper, the coefficients of the series $F_{j, i}(t)$ belong to the completion $\bar{U}_{F}$ of the algebra $U_{F}$.

The completion $\bar{U}_{F}$ determines analyticity properties of products of currents (and coincide with analytical properties of their matrix coefficients for highest weight representations [5]). One can show that for $|i-j|>1$, the product $F_{i}(t) F_{j}(w)$ is an expansion of a function analytic at $t \neq 0, w \neq 0$. The situation is more delicate for $j=i, i \pm 1$. The products $F_{i}(t) F_{i}(w)$ and $F_{i}(t) F_{i+1}(w)$ are expansions of analytic functions at $|w|<\left|q^{2} t\right|$, while the product $F_{i}(t) F_{i-1}(w)$ is an expansion of an analytic function at $|w|<|t|$. Moreover, the only singularity of the corresponding functions in the whole region $t \neq 0, w \neq 0$, are simple poles at the respective hyperplanes, $w=q^{2} t$ for $j=i, i+1$, and $w=t$ for $j=i-1$. Recall, that the deformation parameter $q$ is a generic complex number, which is neither 0 nor a root of unity.

The definition of the composed currents may be written in analytical form

$$
F_{j, i}(t)=-\operatorname{res}_{w=t} F_{j, a}(t) F_{a, i}(w) \frac{d w}{w}=\operatorname{res}_{w=t} F_{j, a}(w) F_{a, i}(t) \frac{d w}{w}
$$


for any $a=i+1, \ldots, j-1$. It is equivalent to the relation

$$
\begin{aligned}
& F_{j, i}(t)=\oint F_{j, a}(t) F_{a, i}(w) \frac{d w}{w}-\oint \frac{q^{-1}-q t / w}{1-t / w} F_{a, i}(w) F_{j, a}(t) \frac{d w}{w}, \\
& F_{j, i}(t)=\oint F_{j, a}(w) F_{a, i}(t) \frac{d w}{w}-\oint \frac{q^{-1}-q w / t}{1-w / t} F_{a, i}(t) F_{j, a}(w) \frac{d w}{w} .
\end{aligned}
$$

In (A.4) $\oint \frac{d w}{w} g(w)=g_{0}$ for any formal series $g(w)=\sum_{n \in \mathbb{Z}} g_{n} z^{-n}$.

Using the relations (A.1) on $F_{i}(t)$ we can calculate the residues in (A.3) and obtain the following expressions for $F_{j, i}(t), i<j$ :

$$
F_{j, i}(t)=\left(q-q^{-1}\right)^{j-i-1} F_{i}(t) F_{i+1}(t) \cdots F_{j-1}(t) .
$$

For example, $F_{i+1, i}(t)=F_{i}(t)$, and $F_{i+2, i}(t)=\left(q-q^{-1}\right) F_{i}(t) F_{i+1}(t)$. The last product is welldefined according to the analyticity properties of the product $F_{i}(t) F_{i+1}(w)$, described above. In a similar way, one can show inductively that the product in the right hand side of (A.5) makes sense for any $i<j$. Formulas (A.5) prove that the defining relations for the composed currents (A.3) or (A.4) yields the same answers for all possible values $i<a<j$.

Calculating formal integrals in (A.4) we obtain the following presentations for the composed currents:

$$
\begin{aligned}
& F_{j, i}(t)=F_{j, a}(t) F_{a, i}[0]-q^{-1} F_{a, i}[0] F_{j, a}(t)+\left(q-q^{-1}\right) \sum_{k<0} F_{a, i}[k] F_{j, a}(t) t^{-k}, \\
& F_{j, i}(t)=F_{j, a}[0] F_{a, i}(t)-q F_{a, i}(t) F_{j, a}[0]+\left(q-q^{-1}\right) \sum_{k \geq 0} F_{a, i}(t) F_{j, a}[k] t^{-k},
\end{aligned}
$$

which are useful for the calculation of their projections.

\section{Acknowledgements}

The main idea to use generating series for the description of the hierarchical Bethe ansatz appeared during authors visit to Max-Planck Institute für Mathematik, Bonn, in January, 2008. Authors acknowledge this scientific center for the hospitality and stimulating scientific atmosphere.

This work was partially done when the second author (S.P.) visited Laboratoire d'Annecy-LeVieux de Physique Théorique in 2006 and 2007. These visits were possible due to the financial support of the CNRS-Russia exchange program on mathematical physics. He thanks LAPTH for the hospitality and stimulating scientific atmosphere. Authors are grateful to Luc Frappat and Éric Ragoucy for many helpful discussions. The authors were supported in part by RFBR grant 08-01-00392 and grant for the support of scientific schools NSh-3036.2008.2. The first author was also supported by the Atomic Energy Agency of the Russian Federation, and by the ANR grant 05-BLAN-0029-01. The second author was also supported in part by RFBR-CNRS grant 07-02-92166-CNRS.

\section{References}

[1] Arnaudon D., Crampè N., Doikou A., Frappat L., Ragoucy E., Spectrum and Bethe ansatz equations for the $U_{q}(g l(\mathcal{N}))$ closed and open spin chains in any representation, Ann. Henri Poincaré 7 (2006), 1217-1268, math-ph/0512037.

[2] Drinfel'd V.G., New realization of Yangians and quantum affine algebras, Soviet Math. Dokl. 36 (1988), 212-216. 
[3] Ding J.T., Frenkel I.B., Isomorphism of two realizations of quantum affine algebra $U_{q}\left(\widehat{\mathfrak{g l}}_{N}\right)$, Comm. Math. Phys. 156 (1993), 277-300.

[4] Ding J., Khoroshkin S., Weyl group extension of quantized current algebras, Transform. Groups 5 (2000), 35-59, math.QA/9804139.

[5] Enriquez B., On correlation functions of Drinfeld currents and shuffle algebras, Transform. Groups 5 (2000), 111-120, math.QA/9809036.

[6] Enriquez B., Khoroshkin S., Pakuliak S., Weight functions and Drinfeld currents, Comm. Math. Phys. 276 (2007), 691-725, math.QA/0610398.

[7] Enriquez B., Rubtsov V., Quasi-Hopf algebras associated with $\mathfrak{s l}_{2}$ and complex curves, Israel J. Math. 112 (1999), 61-108, q-alg/9608005.

[8] Frappat L., Khoroshkin S., Pakuliak S., Ragoucy É., Bethe ansatz for the universal weight function, arXiv:0810.3135.

[9] Khoroshkin S., Pakuliak S., The weight function for the quantum affine algebra $U_{q}\left(\widehat{\mathfrak{s l}}_{3}\right)$, Theor. and Math. Phys. 145 (2005), 1373-1399, math.QA/0610433.

[10] Khoroshkin S., Pakuliak S., Tarasov V., Off-shell Bethe vectors and Drinfeld currents, J. Geom. Phys. 57 (2007), 1713-1732, math.QA/0610517.

[11] Khoroshkin S., Pakuliak S., A computation of an universal weight function for the quantum affine algebra $U_{q}\left(\widehat{g l}_{N}\right)$, J. Math. Kyoto Univ. 48 (2008), 277-322, arXiv:0711.2819.

[12] Kulish P., Reshetikhin N., Diagonalization of $G L(N)$ invariant transfer matrices and quantum $N$-wave system (Lee model), J. Phys. A: Math. Gen. 16 (1983), L591-L596.

[13] Mukhin E., Tarasov V., Varchenko A., Bethe eigenvectors of higher transfer matrices, J. Stat. Mech. Theory Exp. 2006 (2006), no. 8, P08002, 44 pages, math.QA/0605015.

[14] Oskin A., Pakuliak S., Silantyev A., On the universal weight function for the quantum affine algebra $U_{q}\left(\widehat{\mathfrak{g l}}_{N}\right)$, arXiv:0711.2821.

[15] Reshetikhin N., Semenov-Tian-Shansky M., Central extentions of quantum current groups, Lett. Math. Phys. 19 (1990), 133-142.

[16] Tarasov V., Varchenko A., Jackson integrals for the solutions to Knizhnik-Zamolodchikov equation, St. Petersburg Math. J. 2 (1995), no. 2, 275-313.

[17] Tarasov V., Varchenko A., Geometry of $q$-hypergeometric functions, quantum affine algebras and elliptic quantum groups, Astérisque 246 (1997), 1-135, q-alg/9703044.

[18] Tarasov V., Varchenko A., Combinatorial formulae for nested Bethe vectors, math.QA/0702277. 